\title{
Constraining Inflation
}

\author{
Peter Adshead and Richard Easther \\ Department of Physics, Yale University, New Haven, CT 06520, USA
}

\begin{abstract}
Slow roll reconstruction is derived from the Hamilton-Jacobi formulation of inflationary dynamics. It automatically includes information from sub-leading terms in slow roll, and facilitates the inclusion of priors based on the duration on inflation. We show that at low inflationary scales the Hamilton-Jacobi equations simplify considerably. We provide a new classification scheme for inflationary models, based solely on the number of parameters needed to specify the potential, and provide forecasts for likely bounds on the slow roll parameters from future datasets. A minimal running of the spectral index, induced solely by the first two slow roll parameters ( $\epsilon$ and $\eta$ ) appears to be effectively undetectable by realistic Cosmic Microwave Background experiments. However, we show that the ability to detect this signal increases with the lever arm in comoving wavenumber, and we conjecture that high redshift $21 \mathrm{~cm}$ data may allow tests of second order consistency conditions on inflation. Finally, we point out that the second order corrections to the spectral index are correlated with the inflationary scale, and thus the amplitude of the CMB B-mode.
\end{abstract}




\section{Introduction}

Inflation is an elegant explanation for the large scale appearance of our universe. Causally connected regions of space are swept outside the Hubble horizon during a phase of accelerated expansion and cross back during a later epoch of regular expansion. The inflationary predictions of a flat universe, along with a Gaussian, adiabatic and nearly scale invariant power spectrum are perfectly consistent with current data. Moreover, the observed anti-correlation between temperature and polarization at large scales provides further support for the inflationary origin of the initial density perturbations.

While inflation predicts the overall form of our visible universe, we have very little understanding of the physical mechanism that generates the accelerated expansion. Consequently, there is considerable interest in "reverse engineering" the inflationary potential from astrophysical data. The primordial power spectrum can be written as a function of the slow roll parameters. In their simplest form, the slow roll parameters are expressed as derivatives of the potential, so measuring the spectrum to arbitrary precision would yield a Taylor expansion of the potential. Unfortunately, the simplicity of this scheme is undermined by the practical challenges that arise during its implementation. Recall that the evidence for any scale dependence in the primordial spectrum is still preliminary, although there is indeed growing evidence the spectrum is red, or has diminishing power at short scales [1]. Reconstructing the potential requires reliably distinguishing between models which all predict slightly different red spectra, which would need an improvement in the accuracy of cosmological parameter determinations by a further order of magnitude. Given both cosmic variance and the practical challenges of foreground subtraction it is not clear that this is possible, even in principle. This is a particularly pressing problem if we are limited to data derived from the CMB [Cosmic Microwave Background] and LSS [Large Scale Structure]. These two sources of data are sensitive to a range of scales that differ by a total factor of perhaps $10^{4}$ but in simple models of inflation the primordial spectrum changes very slowly, undermining the power of any reconstruction program. Despite these challenges, there are powerful motivations for theoretical studies of reconstruction. The first is that the physical basis of inflation (if inflation is, in fact, the source of the primordial fluctuations) is one of the most important open questions in all of cosmology, and answering it is likely to shed light upon particle physics at very high energies. Secondly, a number of proposed experiments aim to measure the fundamental spectrum to very high precision; we wish to determine their ability to constrain the overall inflationary parameter space, even though many of them will take decades rather than years to implement.

The reconstruction of the inflationary potential was was first discussed in the early 1990s [2, 3, 4, 5]. This paper builds on the slow roll reconstruction algorithm, proposed and implemented by Easther and Peiris [6, 7, 8], which grew out of a Monte Carlo approach based on the inflationary flow equations [9, 10, 11, 12, 13]. Originally reconstruction was based on taking the measured values of the spectral indices (and their running), solving for the slow roll parameters, and deducing the form of the potential 
[14. Since we are interested in the inflationary parameter space, there is no need to compute the spectral indices, as these have no fundamental significance. Rather, one can include the slow roll variables directly in the cosmological parameter set and bound them using Monte Carlo Markov Chain fits to cosmological data [15, 6, 7]. Slow roll reconstruction is thus an optimal approach to recovering the inflationary potential from data, since it makes use of all available information. Moreover, while it makes use of the slow roll expansion, we need never employ the slow roll approximation, as the truncated Hamilton-Jacobi hierarchy can be solved exactly [16]. Slow roll reconstruction thus captures all the correlations between the slow roll parameters, including those at second and higher order $\ddagger$

A number of other approaches to reconstruction have been proposed. In particular, Leach and collaborators write the spectral indices (and their running) in terms of the slow roll parameters at a fixed pivot [19, 20, 21]. When used with data which probes a small range of scales, this approach is functionally identical to slow roll reconstruction, since the slow roll parameters are effectively constant. However, we will show that as the lever arm in wavelength becomes large, the scale dependence of these parameters can be significant. In principle, one could account for this running by computing the perturbation spectrum using expressions that include higher order corrections in slow roll, but this approach will become algebraically cumbersome at some point. Conversely, [22] explores constraints on the slow roll parameters imposed by demanding a sufficient period of inflation. Finally Cline and Hoi look at reconstructing inflationary models with significant running within the Hamilton-Jacobi formalism [23. Since slow roll reconstruction implicitly predicts the form of the potential it naturally makes use of this information, and one can restrict fits to parameter values that allow a sufficiently long period of inflation.

The aim of this paper is to forecast the parameter constraints we can expect from slow roll reconstruction when it is applied to future datasets, and to explore the differences between slow roll reconstruction and fits to the usual spectral parameters. In the process, we show that low scale inflation (i.e. energies significantly below $10^{15} \mathrm{GeV}$ ) is described by a simplified set of Hamilton-Jacobi equations, as the first parameter $(\epsilon)$ is effectively absent from the dynamical system. We present a new classification scheme for inflationary models based on the number of free parameters needed to specify the potential - rather than its shape - and show this is naturally related to the (truncated) slow roll hierarchy. We then use Fisher matrix calculations [24, 25] to explore how constraints on the inflationary parameter space improve with the lever arm in wavelength probed by the dataset \$ With a purely Gaussian likelihood function $\ddagger$ As explicitly implemented in [6, 7, slow roll reconstruction uses approximate expressions for the perturbation spectrum, but this is simply a matter of convenience [6]. One can always solve the perturbation mode equations numerically, and this approach has been explored [17, 18]. As we will see, computing the spectrum with the scale dependent slow roll parameters yields a very good match to the exact calculation. Numerically evaluated spectra eliminate a source of uncertainty in the parameter constraints, but are not justified by the quality of presently available data.

$\S$ We utilize CAMB, http://camb.info/, [26] for calculations of the CMB spectra and the matter power 
(which is an assumption of the Fisher matrix formalism) the CMB alone does not probe a large enough range of wavelengths to produce results that differ significantly from those obtained via fits to the standard spectral parameters. However, if one has access to the primordial spectrum at very short scales (e.g. via high redshift $21 \mathrm{~cm}$ measurements), terms that are second order in slow roll may become significant. In practice, the likelihood is far from Gaussian, so this analysis is effectively a worst case scenario. In particular, slow roll reconstruction allows us to include information about the duration of inflation into parameter estimates; these constraints are not captured by a Fisher matrix analysis. Models with a significant tensor spectrum generically require a longer period of inflation than those with an unobservable tensor component; we show how this information - along with a very mild prior on the post-inflationary equation of state - can give further leverage to slow roll reconstruction.

Since we are considering constraints on the inflationary parameter space, we are primarily interested in models for which $\Omega_{\text {Tot }} \equiv 1$; the flatness of the spatial hypersurfaces is a key prediction of inflation. This is an implicit assumption of slow roll reconstruction, since the Hamilton-Jacobi equations are derived after ignoring the spatial curvature term in the Einstein equations. If $\Omega_{\text {Tot }}$ differs slightly from unity today the longest modes will have left the horizon just as inflation began. Consequently, slow roll reconstruction (as currently implemented) is only self-consistent when inflation lasts long enough to suppress any transients associated with the pre-inflationary initial conditions before modes which contribute to the quadrupole leave the horizon. We also assume that the entire primordial perturbation spectrum was generated during inflation, and contains no significant contribution from cosmic strings, or other non-inflationary mechanisms. Finally, we treat the dark energy as a pure cosmological constant, although relaxing this assumption would not significantly modify our key conclusions.

The CMB temperature peak morphology is very well understood [28, 29, 30, 31, 32], along with the importance of the $E$ and $B$ polarization modes. The $\ell$ th multipole of the CMB anisotropy corresponds to a wavenumber $k^{-1} \simeq 2 /\left(H_{0} \ell\right)$, so the CMB probes scales from $k \sim 2 \times 10^{-4} h \mathrm{Mpc}^{-1}$ at the quadrupole to $k \sim 0.18 h \mathrm{Mpc}^{-1}$ at $\ell \sim 1500$. Beyond $\ell \sim 1500$, the primordial $C_{\ell}$ decay sharply due to Silk damping, which reflects the finite width of the surface of last scattering. It is thus difficult to measure the primordial CMB at shorter scales. Moreover, foregrounds and secondary anisotropies typically grow in amplitude at smaller scales. In what follows, we will consider perfect CMB measurements out to $\ell_{\text {MAx }}$ of up to 2,500 in order to explore the lever arm yielded by data over a large range of angular scales. However, given the difficulties associated with foreground subtraction, these calculations are essentially gedanken experiments.

On the other hand, we are not limited to CMB information alone. The information contained in LSS data is largely orthogonal to that in the CMB, breaking many parameter degeneracies. This 'cosmic complementarity' is well known [33, 34, 27, 35], and the slow roll reconstruction can obviously make use of this data. While the spectrum, and the two sided derivative methodology outlined in [27]. 
linear regime of structure formation is very well understood, recovering the primordial spectrum at length scales which have undergone nonlinear evolution is a challenging task. The smallest scale still in the linear regime probed by low low-redshift LSS experiments is not wildly different from that probed by high resolution measurements of the CMB. In what follows, we are primarily interested in the differences between slow roll reconstruction and analyses based on the usual spectral variables, and adding LSS data would improve both approaches. Furthermore, forecasts of parameter uncertainties for LSS data depend significantly on the design of the experiment. Consequently, in this analysis we have focussed on $\mathrm{CMB}$ data alone. One can probe smaller scales by looking at very high redshift data, when these modes were still in the linear regime. Options in this area include very deep galaxy surveys, or Lyman- $\alpha$ experiments. More speculatively - but with far greater potential power - the high redshift $21 \mathrm{~cm}$ background may allow probes of the primordial spectrum at very small wavelengths [36]. We plan to look at constraints derived from combined fits to $\mathrm{CMB}$ and $21 \mathrm{~cm}$ data in a future paper, but the calculations here suggest that such a measurement might permit tests of higher order inflationary consistency conditions [37, 38, 14. Finally, a BBO style experiment is sensitive to the primordial tensor spectrum at solar system scales, and slow roll reconstruction could take advantage of this data to put exquisitely accurate constraints on the inflationary potential.

This paper is organized as follows. In $\$ 2$ we review inflationary dynamics, the slow roll approximation and our construction of the primordial spectrum. In $\$ 3$ we consider analytic models with low inflationary scales, and construct a new classification scheme for inflationary models in $\$ 4$, In $\$ 5$ we review the Fisher matrix formalism for CMB anisotropy experiments, and describe the resulting forecasts in $\$ 6$. We conclude in $\$ 7$. Finally, in an Appendix we give a more detailed analysis of the slow roll dynamics at low inflationary scales.

\section{Inflation and the Primordial Power Spectrum}

\subsection{The Background}

We use the Hamilton-Jacobi formulation of inflationary dynamics, expressing the Hubble parameter as a function of $\phi$, rather than as a function of time. Thus $H \equiv H(\phi)$, and we assume that $\phi$ is monotonic. The equations of motion are [14, 39, 40, 41, 42, 43]

$$
\begin{aligned}
& \dot{\phi}=-\frac{m_{\mathrm{Pl}}^{2}}{4 \pi} H^{\prime}(\phi), \\
& {\left[H^{\prime}(\phi)\right]^{2}-\frac{12 \pi}{m_{\mathrm{Pl}}^{2}} H^{2}(\phi)=-\frac{32 \pi^{2}}{m_{\mathrm{Pl}}^{4}} V(\phi) .}
\end{aligned}
$$

Primes denote derivatives with respect to the field, while an overdot denotes derivatives with respect to coordinate time. Equation (2) is the Hamilton-Jacobi equation, and describes inflation in terms of the Hubble parameter, $H(\phi)$, rather than the potential, $V(\phi)$. The Hubble parameter, being a geometric quantity, describes the spacetime 
dynamics, whereas particle physics constructions predict $V(\phi)$. We can thus discuss slow roll inflation without specifying the particle physics that generates inflation. The HSR [Hubble slow roll] parameters ${ }^{\ell} \lambda_{H}$ are defined by the infinite hierarchy of differential equations [10]:

$$
\begin{aligned}
\epsilon(\phi) & \equiv \frac{m_{\mathrm{Pl}}^{2}}{4 \pi}\left[\frac{H^{\prime}(\phi)}{H(\phi)}\right]^{2}, \\
{ }^{\ell} \lambda_{H} & \equiv\left(\frac{m_{\mathrm{Pl}}^{2}}{4 \pi}\right)^{\ell} \frac{\left(H^{\prime}\right)^{\ell-1}}{H^{\ell}} \frac{d^{\ell+1} H}{d \phi^{(\ell+1)}} ; \ell \geq 1 .
\end{aligned}
$$

The usual slow roll parameters are $\eta={ }^{1} \lambda_{H}$ and $\xi={ }^{2} \lambda_{H}$. If we truncate the hierarchy, so that ${ }^{\ell} \lambda_{H}=0$ for all $\ell>M$ at some $\phi_{0}$, then these ${ }^{\ell} \lambda_{H}$ vanish everywhere. When truncated at order $M$, the hierarchy can be solved exactly to obtain [16]

$$
\frac{H(\phi)}{H_{0}}=\sum_{n=0}^{M+1} B_{n}\left(\frac{\phi}{m_{\mathrm{Pl}}}\right)^{n},
$$

where the $B_{n}$ are specified by the initial values of the HSR parameters:

$$
B_{0}=1, \quad B_{1}=\sqrt{4 \pi \epsilon_{0}}, \quad B_{\ell+1}=\frac{(4 \pi)^{\ell}}{(\ell+1) ! B_{1}^{\ell-1}} \lambda_{H, 0} .
$$

The subscript 0 refers to their value at the moment the fiducial mode $k_{0}$ leaves the horizon (when $a H=k_{0}$ ) and $\phi=\phi_{0}=0$. In this analysis we set $k_{0}=0.05 \mathrm{Mpc}^{-1}$, which corresponds to $\ell \sim 500$ in the CMB.

Substituting equation (3) into equation (2) gives the potential

$$
V(\phi)=\frac{3 m_{\mathrm{Pl}}^{4}}{8 \pi^{2}} H^{2}(\phi)\left[1-\frac{1}{3} \epsilon(\phi)\right],
$$

while $N$, the number of e-folds before the end of inflation is

$$
\frac{d N}{d \phi}=\frac{4 \pi}{m_{\mathrm{Pl}}^{2}} \frac{H}{H^{\prime}}=\frac{2 \sqrt{\pi}}{m_{\mathrm{Pl}}} \frac{1}{\sqrt{\epsilon(\phi)}},
$$

and $\phi$ and $k$ are related by

$$
\frac{d \phi}{d \ln k}=-\frac{m_{\mathrm{Pl}}}{2 \sqrt{\pi}} \frac{\sqrt{\epsilon}}{1-\epsilon} .
$$

The scale dependence of the slow roll parameters follows from equations (3) and (8) :

$$
\begin{aligned}
& \frac{d \epsilon}{d N}=2 \epsilon(\eta-\epsilon), \\
& \frac{d \eta}{d N}=-\epsilon \eta+\xi, \\
& \frac{d^{\ell} \lambda_{H}}{d N}=[(\ell-1) \eta-\ell \epsilon]^{\ell} \lambda_{H}+{ }^{\ell+1} \lambda_{H},
\end{aligned}
$$

and the truncation property mentioned above can be derived from the last equation. Note that these formulae use $N$ as their independent variable.

Once we have specified the values of the slow roll parameters at some fiducial scale, we may "flow" to any other scale by using the slow roll hierarchy. The scale dependence 
of the slow roll parameters ensures that any non-trivial correlation between the slow roll parameters will only strictly apply at a single value of $\phi$. For instance, if might assume the existence of a special point where $\epsilon$ and $\eta$ were non-zero, while ${ }^{i} \lambda_{H}=0$ for $i=3, \cdots, N-1$ and ${ }^{N} \lambda_{H} \neq 0$ (see [44] for an example). In principle, one could analyze this type of model by adding $k_{0}$ to the parameter set in order to marginalize over the model-dependence in the mapping between $k$ and $\phi$.

\subsection{The Perturbations}

The scalar and tensor perturbations obey [45],

$$
\begin{aligned}
& \frac{d^{2} u_{k}}{d \tau^{2}}+\left(k^{2}-\frac{1}{z} \frac{d^{2} z}{d \tau^{2}}\right) u_{k}=0, \\
& \frac{d^{2} v_{k}}{d \tau^{2}}+\left(k^{2}-\frac{1}{z} \frac{d^{2} z}{d \tau^{2}}\right) v_{k}=0 .
\end{aligned}
$$

Here $\tau$ is conformal time, $u_{k}$ are the Fourier modes of the gauge invariant Mukhanov potential describing the intrinsic curvature perturbation, $v_{k}$ are the analogous Fourier modes for the tensor perturbations, while $z=a \dot{\phi} / H$ for scalar perturbations, and $z=a$ for tensor perturbations. The power spectra are

$$
\begin{aligned}
& \mathcal{P}_{\mathcal{R}}=\frac{k^{3}}{2 \pi^{2}}\left|\frac{u_{k}}{z}\right|^{2}, \\
& \mathcal{P}_{h}=\frac{32 k^{3}}{\pi m_{\mathrm{pl}}^{2}}\left|\frac{v_{k}}{a}\right|^{2} .
\end{aligned}
$$

A first order expansion about the exact solution for power law inflation gives [46, 6, 7]

$$
\begin{aligned}
& \mathcal{P}_{\mathcal{R}}=\left.\frac{[1-(2 C+1) \epsilon+C \eta]^{2}}{\pi \epsilon}\left(\frac{H}{m_{\mathrm{Pl}}}\right)^{2}\right|_{k=a H}, \\
& \mathcal{P}_{h}=\left.[1-(C+1) \epsilon]^{2} \frac{16}{\pi}\left(\frac{H}{m_{\mathrm{Pl}}}\right)^{2}\right|_{k=a H},
\end{aligned}
$$

where $C=-2+\ln 2+\gamma \approx-0.729$ and $\gamma$ is the Euler-Mascheroni constant. The power spectrum is normalized at $k_{0}$ by setting

$$
A_{s}=\frac{\left[1-(2 C+1) \epsilon_{0}+C \eta_{0}\right]^{2}}{\pi \epsilon_{0}}\left(\frac{H_{0}}{m_{\mathrm{Pl}}}\right)^{2}
$$

where $\epsilon_{0}, \eta_{0}$ and $H_{0}$ are the values of these parameters at $k_{0}$.

One can always solve the perturbation evolution equations numerically [47, 48, 49, 50]. This approach is implemented in [17, 18] who parametrize $H(\phi)$ as a polynomial of finite order - which is identical to slow roll reconstruction, $\|$ since the truncated flow

\| Note that [17, 18] also consider fits to a polynomial expression for $H^{2}(\phi)$. Hamann, Lesgourgues, and Valkenburg [18] present constraints on the first three slow roll parameters from WMAP3 [51] and ACBAR [52] derived with two different approximations to the spectrum, as well as the numerically evaluated mode equations. Their analysis shows that the difference between these results is entirely explained by the implicit priors on the duration of inflation, and not the accuracy with which $P(k)$ is evaluated. The ability to include constraints based on the duration of inflation is a key feature of slow roll reconstruction, and we return to this topic below. 

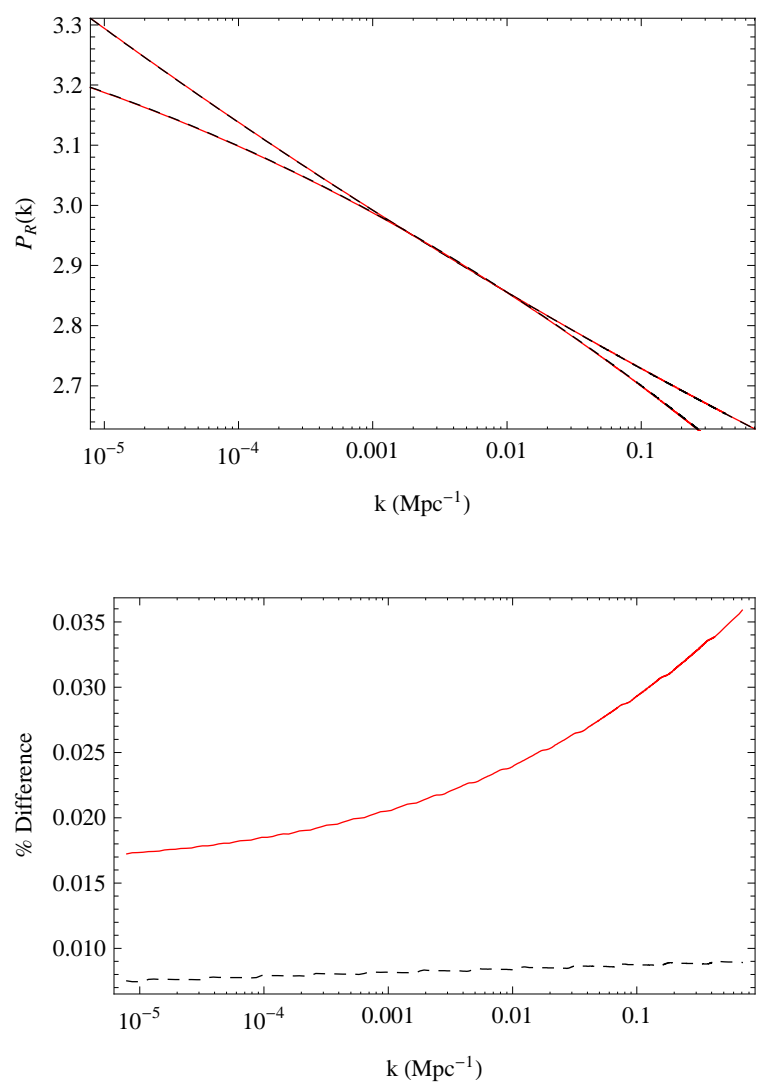

Figure 1. The top panel shows the primordial spectra for two parameter choices; $\epsilon=0.01, \eta=0.01, \xi=0$ (upper) and $\epsilon=0.01, \eta=0.01, \xi=0.001$ (lower, curved). The bottom panel shows the difference between the spectra, the dashed curve applies to $\xi=0.0$ while the solid curve corresponds to $\xi=0.001$. For extreme values of $\xi$ these discrepancies can become large, but these models typically have a very low number of e-folds.

hierarchy is solved exactly by a polynomial in $H(\phi)$ [15]. Since equation (17) uses the scale dependent slow roll parameters - which are matched to a value of $k$ by solving equation (9) - it accurately tracks the exact spectrum for the combinations of slow roll parameters one is likely to encounter in practice. We plot the difference between equation (17) and the exact spectrum for two representative sets of slow roll parameters in Figure 1. In this paper, we perform our calculations using equations (17) and (18), but a numerically computed spectrum would banish the last vestiges of the slow roll approximation from this analysis, and might be justified when dealing with very high quality data, especially if the running in the spectral index turns out to be non-trivial.

From (17) and (18), one can also recover expressions for the usual spectral indices and their scale dependence [14]:

$$
\begin{array}{ll}
n_{s} & =1+2 \eta-4 \epsilon-2(1+\mathcal{C}) \epsilon^{2}-\frac{1}{2}(3-5 \mathcal{C}) \epsilon \eta+\frac{1}{2}(3-\mathcal{C}) \xi, \\
r & =16 \epsilon[1+2 C(\epsilon-\eta)],
\end{array}
$$




$$
\begin{aligned}
\frac{d n_{s}}{d \ln k}= & -\frac{1}{1-\epsilon}\left\{2 \frac{d \eta}{d N}-4 \frac{d \epsilon}{d N}-4(1+\mathcal{C}) \epsilon \frac{d \epsilon}{d N}-\right. \\
& \left.\frac{1}{2}(3-5 \mathcal{C})\left(\epsilon \frac{d \eta}{d N}+\eta \frac{d \epsilon}{d N}\right)+\frac{1}{2}(3-\mathcal{C}) \frac{d \xi}{d N}\right\}, \\
n_{t}= & -2 \epsilon-(3+\mathcal{C}) \epsilon^{2}+(1+\mathcal{C}) \epsilon \eta,
\end{aligned}
$$

where $\mathcal{C}=4(\gamma+\ln 2)-5$ and is not to be confused with $C$ above. The expressions are not used during slow roll reconstruction, but are employed when we make comparisons to the empirical characterization.

\subsection{The Duration of Inflation}

We need make no explicit assumption about the duration of inflation. Other HSR based analyses often pick a specific set of parameters, then integrate forward in time to find the moment at which inflation ends, and then work backwards from this point to find the slow roll parameters some fixed number of e-folds before the end of inflation [10, 11, 12, 13. By running Markov Chains with the slow roll variables in the parameter set we learn their values at the moment when cosmological scales were leaving the horizon. We can then evolve them forwards in time, to discover the point (if any) at which inflation ends, which occurs when $\epsilon=1$. In three parameter fits to current data, one finds considerable support for a running index, which requires large values of $\xi$, leading to models with $N \sim 15$ or even less [7, 8]. If we assume that we have enough slow roll parameters to describe the inflationary potential this situation is clearly not self-consistent. One can posit the existence of a secondary period of inflation, or expand the parameter set in order to make $\xi$ strongly scale-dependent, but either of these "solutions" implies that the set $\{\epsilon, \eta, \xi\}$ is incomplete. Conversely, if we find a large or even unbounded number of e-folds for a given set of slow roll parameters, the implication is that inflation ends via some sort of hybrid or waterfall transition [53, 54], where the field evolves in a direction orthogonal to the original inflaton trajectory. These two scenarios are analogous to following a path that leads down a mountainside to a valley or walking along a gentle plain and then encountering a sharp cliff, respectively. In the former case, the end of inflation can be extrapolated from the slope of the potential some distance away from the minimum, while in the latter case the inflaton field receives little or no advance warning that inflation is about to end.

The primordial tensor amplitude is set by the energy scale of inflation. Assuming slow roll, the scalar amplitude is given by 55

$$
\mathcal{P}_{S, 0}=\frac{8 V_{k}}{3 m_{\mathrm{Pl}}} \frac{1}{\epsilon}
$$

where $\mathcal{P}_{S, 0} \simeq 2.6 \times 10^{-9}\left[20\right.$ ] and $V_{k}$ is the value of the potential as the mode $k$ leaves

the horizon. This energy scale can be translated into a constraint on the number of e-folds needed to reproduce the observed universe [15]

$$
N(k)=63.3+\frac{1}{4} \ln \epsilon(k)-\ln \left[\frac{k}{a_{0} H_{0}}\right]+\ln \left[\frac{V_{k}^{\frac{1}{4}}}{V_{\text {end }}^{\frac{1}{4}}}\right]-\frac{1}{3} \ln \left[\frac{V_{\text {end }}^{\frac{1}{4}}}{\rho_{\text {reh }}^{\frac{1}{4}}}\right],
$$


where $V_{\text {end }}$ is the energy scale at the end of inflation, $\rho_{\text {reh }}$ is the energy density at reheating and $a_{0} H_{0}$ is the expansion rate today. As pointed out by Kinney and Riotto [56], the undetermined parameters in $N(k)$ induce a theoretical uncertainty in associating inflationary parameters with a potential.

By writing down equation (25) we have assumed that the universe is matter dominated after the end of inflation and then radiation dominated between the end of reheating and matter-radiation equality, which amount to assuming that the effective equation of state parameter varies between $w=0$ and $w=1 / 3$ between the of inflation and nucleosynthesis. If we include matter with $w>1 / 3$, such as a kination field [57], $N(k)$ will increase. The minimum possible value of $w$ in a decelerating universe is $-1 / 3$, and if $-1 / 3<w<0, N(k)$ can be substantially reduced. Note that the term involving $V_{k}$ implicitly contains $\epsilon$. However, in slow roll inflation $V_{k}$ is not expected to differ considerably from $V_{\text {end }}$, and thus this term is not considered to be important.

Slow roll reconstruction allows one to include priors based on the duration of inflation when fitting to the slow roll parameters. In particular, the duration of inflation depends very strongly on the value of $\xi[8$, and even the modest requirement that $N>30$ induces stringent bounds on the parameter space [6]. However, studying equations (24) and (25) shows that the value of $N$ depends strongly on $\epsilon$ - while we can certainly have inflationary models with $N \sim 30$, this requires a tiny inflationary scale, and thus a miniscule value of $\epsilon$. Conversely, if $\epsilon \sim 0.01$, the inflationary scale must be comparatively large, and $N \sim 50$. Consequently, we can implement slow roll reconstruction with an $\epsilon$ dependent constraint on the number of e-folds by using equation (24) to write $V_{k}$ as a function of $\epsilon$ and the amplitude of the primordial perturbation spectrum. This requires a mild prior on the post-inflationary equation of state - for instance that $w>0$ after the end of inflation. This implicitly rules out exotic scenarios such as a phase in which the universe is dominated by a frustrated network of cosmic strings [58], but does not strongly constrain the post-inflationary universe. Finally, a principal lesson of [18] is that all implementations of slow roll reconstruction make implicit assumptions about the minimal permissible duration of inflation. Consequently, slow roll reconstruction is most transparent when an explicit prior on $N$ is included in the chains, even if that bound is very mild, and we will pursue this topic in a separate paper.

\section{Slow Roll in the Low- $\epsilon$ limit}

A primordial gravitational wave spectrum is often described as the "smoking gun" of inflation, since there is no credible alternative mechanism for generating long wavelength gravitational waves. Detecting this signal would determine the overall energy scale of inflation since $\mathcal{P}_{h} \sim H^{2}$. Intriguingly, string theoretic inflationary scenarios generically predict a negligible amount of primordial gravitational radiation [59], or $r<10^{-10}$. In this case, $\epsilon$ must also be tiny, since $r \sim 16 \epsilon$. Conversely, models with algebraically simple potentials typically have $r>0.001$ 60]. The constraint on stringy models can 
be understood in terms of the Lyth bound [61], which requires that the inflaton's total excursion be sub-Planckian to ensure that its potential is not dominated by contributions from higher-order operators.

The current observational bound on $r$ is relatively weak, $r<0.3$ [51], but upcoming CMB experiments may push this value down to $r \sim 0.01$ [62, 63, 64]. However, if $r$ is many orders of magnitude smaller than unity it may be unobservable by any conceivable experiment [65]. While $\epsilon$ must be tiny in these models, there is no equivalent constraint on $\eta$, and in many supergravity or stringy models the challenge is to ensure that $|\eta|<1$. Consequently, we assume $1 \gg|\eta| \gg \epsilon$, and write the flow equations as

$$
\begin{aligned}
& \frac{d \eta}{d N}=\xi \\
& \frac{d \xi}{d N}=\eta \xi+{ }^{3} \lambda_{H}, \\
& \frac{d^{\ell} \lambda_{H}}{d N}=(\ell-1) \eta^{\ell} \lambda_{H}+{ }^{\ell+1} \lambda_{H} .
\end{aligned}
$$

Here $\xi$ drives the evolution of $\eta$ and ${ }^{3} \lambda_{H}$ drives the evolution of $\xi$ (if we could measure it), and one can still truncate the hierarchy. We can also estimate $\epsilon$,

$$
\frac{d \epsilon}{d N}=2 \epsilon \eta+\mathcal{O}\left(\epsilon^{2}\right)
$$

which can be solved to give

$$
\epsilon(N)=\epsilon\left(N_{0}\right) \exp \left[2 \int_{N_{0}}^{N} \eta(N) d N\right] .
$$

Since $\eta \ll 1$, if $\epsilon$ is initially small, it stays small and then rises super-exponentially as $\eta \sim 1$. Conversely, if $\eta$ is also vanishingly small, we cannot usefully extend this truncation, since $\epsilon$ is unique in that both of its source terms in the slow roll hierarchy are suppressed when it is small. To illustrate, suppose that the first $m-1$ slow roll parameters are close to zero, but ${ }^{m} \lambda_{H} \neq 0$. However, ${ }^{m} \lambda_{H}$ generates ${ }^{n} \lambda_{H} \neq 0$ for all $n<m$, so this condition amounts to choosing the initial conditions at a very special point in the potential. The coupling between ${ }^{m} \lambda_{H}$ and ${ }^{m-1} \lambda_{H}$ means that after one e-fold ${ }^{m-1} \lambda_{H}$ will be at least as large as ${ }^{m} \lambda_{H}$. One may proceed inductively to see that after $m$ e-foldings (and probably sooner) all of the slow roll parameters except for $\epsilon$ will differ significantly from zero.

When we drop $\epsilon$, we can solve the slow roll hierarchy exactly if it is truncated at a relatively low order. These solutions are explored in Appendix A. Consider the specific case $\xi=0, \eta \ll 1$ and $\epsilon \ll \eta$, so $d \eta / d N \approx 0$ and $\eta(N) \approx \eta(0) \equiv \eta_{0}$, which corresponds to the "Low- $\epsilon$ 1-Parameter" model defined in the next section. From equation (22) it follows that $n_{s}$ is effectively constant over cosmological scales. Equation (10) has the solution

$$
\epsilon(N)=\frac{\eta_{0} \epsilon_{0}}{\epsilon_{0}+\left(\eta_{0}-\epsilon_{0}\right) e^{-2 \eta_{0} N}}
$$




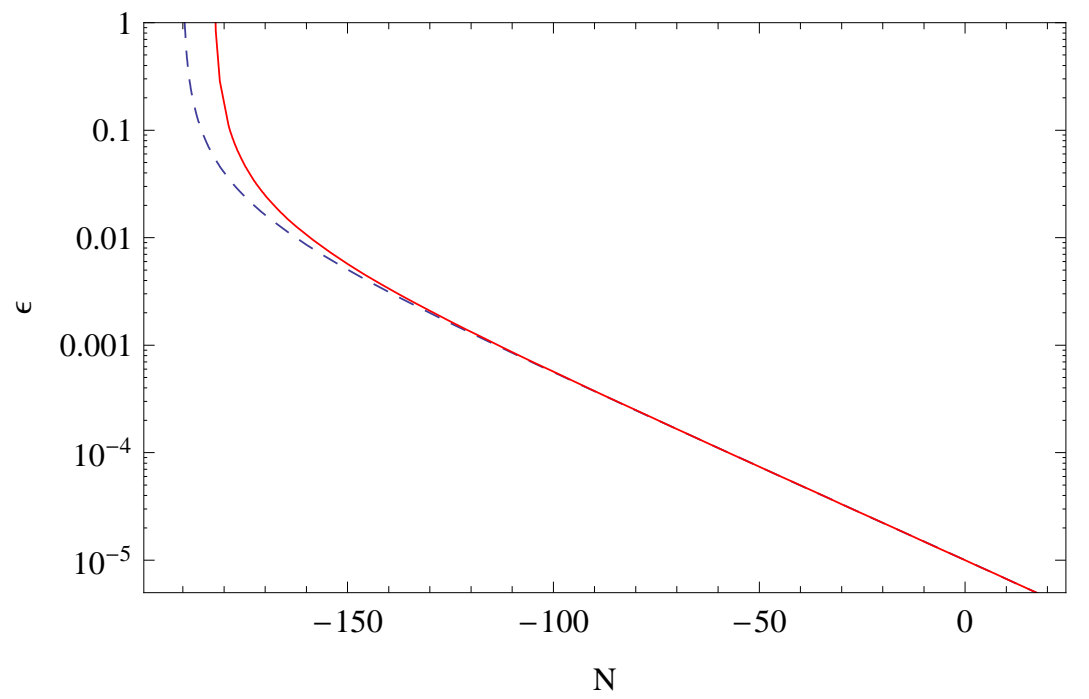

Figure 2. Slow roll hierarchy truncated at $\xi=0$. The plot shows the evolution of $\epsilon$, the red curve is the exact result, the dashed blue curve is the approximation with $\eta$ constant. We have set $\eta=-0.02$ and $\epsilon=10^{-5}$.

where we have set $N=0$ at the fiducial scale for convenience. In this approximation, we can solve exactly for where inflation ends:

$$
N(\epsilon=1)=-\frac{1}{2 \eta_{0}} \ln \left(\frac{\epsilon_{0}\left(\eta_{0}-1\right)}{\eta_{0}-\epsilon_{0}}\right),
$$

and we recall that unless $\eta_{0}<0$, inflation will continue indefinitely in this scenario.

If we have $\epsilon \sim 10^{-10}$, and $\eta=-0.02$ (e.g. $n_{s} \approx 0.96$ ), we have inflation ending $N \sim 500$ e-folds after the fiducial scale has left the horizon while $\epsilon \sim 10^{-5}$ gives a futher $N \sim 190$. As illustrated by Figure 2 , the approximate solution is typically good up until the last couple of e-folds and leads to a slight overestimate of $N$. Consequently, if $\eta \approx-0.02$, and $\epsilon$ is very small, inflation either ends via a hybrid transition when it encounters an abrupt cliff in the potential, or at least one higher order slow roll parameter has a non-trivial value. Further, looking at equation (22), if $\epsilon$ is tiny and ${ }^{\ell} \lambda_{H}=0$ for $\ell>2$ the running is entirely dominated by $\xi$ and is thus negligible if $\xi=0$. Conversely, if $\epsilon \approx 0.01$, some running will be generated by the $\epsilon^{2}$ term in the slow roll hierarchy, even if $\xi=0$.

We also see that $\xi$ quickly dominates the dynamics when it is of the same order of magnitude as $\eta$, as happens when we try to match the central value of the running derived from WMAP data. As noted by [8] the effect of this is to reduce the number of e-folds to an unacceptably small level. If the running is significant, then we would need at least one further slow-roll parameter in order to ensure that $\xi$ itself is scale dependent. Unless such a scenario is a prediction of a well-motivated model, this situation would justifiably be regarded as a fine-tuning. 


\begin{tabular}{|c|c|c|c|}
\hline Class & HSR & Spectral & Shorthand \\
\hline High- $\epsilon$ 1-Parameter & $\epsilon$ & $n_{s}-1=\frac{1}{4} r=-2 n_{t}, \alpha \approx 0$ & - \\
\hline Low- $\epsilon$ 1-Parameter & $\eta$ & $n_{s} \neq 1, r \approx 0, \alpha \approx 0$ & $\Lambda$ CDM \\
\hline High- $\epsilon$ 2-Parameter & $\epsilon, \eta$ & $n_{s}-1 \neq \frac{1}{4} r=-\frac{1}{2} n_{t}, \alpha \approx 0$ & $\Lambda$ CDM $+r$ \\
\hline Low $\epsilon$ 2-Parameter & $\eta, \xi$ & $n_{s} \neq 1, r \approx 0, \alpha \neq 0$ & $\Lambda$ CDM $+\alpha$ \\
\hline High- $\epsilon$ 3-Parameter & $\epsilon, \eta, \xi$ & $n_{s}-1=\frac{1}{4} r=-\frac{1}{2} n_{t}, \alpha \neq 0$ & $\Lambda$ CDM $+r+\alpha$ \\
\hline Low- $\epsilon$ 3-Parameter & $\eta, \xi,{ }^{3} \lambda_{H}$ & $n_{s} \neq 1, r \approx 0, \alpha \neq 0 \ldots$ & - \\
\hline
\end{tabular}

Table 1. We show the non-trivial variables for models with up to three HSR parameters, and the equivalent set of variables written in terms of the scalar and tensor indices. The final column denotes the corresponding shorthand for the resulting model. The canonical $\Lambda \mathrm{CDM}$ case corresponds to assuming that $\epsilon \ll|\eta|$. In this column $r$ refers to adding a tensor spectrum, while $\alpha$ denotes the running of the spectral index.

\section{Parameter Counting and Model Classification}

We now describe a classification scheme for inflationary models, based on the slow roll hierarchy. In the past, it has been tacitly assumed that $\epsilon$ is the one parameter that cannot be dropped from the slow roll hierarchy. Consequently, all previous implementations of slow roll reconstruction have allowed for a tensor component in the $\mathrm{CMB}$, whereas the minimal $\Lambda \mathrm{CDM}$ parameter set includes only scalar perturbations. In the language of slow roll, the $\Lambda \mathrm{CDM}$ parameter set is thus equivalent to assuming that $\epsilon \lesssim 10^{-5}$, in which case $\epsilon$ plays no direct role in determining the observable properties of the present universe, and we need to include it in our chains. We thus propose the following scheme:

- Low- $\epsilon, N$-parameter. The tensor-scalar ratio is assumed to be immeasurably small, and we truncate the slow roll hierarchy at ${ }^{N+1} \lambda$.

- High- $\epsilon, N$-parameter. The tensor-scalar ratio can be measurably different from zero, and we truncate the slow roll hierarchy at ${ }^{N} \lambda$.

This is distinct from the "large field / small field / hybrid" zoology (see e.g. [12]). Models with a large variation in $\phi$ necessarily have a non-trivial $\epsilon$ [61, 66], whereas the small field and hybrid cases are distinguished by the sign of $\eta$. However, when $\xi$ is nontrivial, $\eta$ can change sign during the course of inflation, so "shape-based" taxonomies are best avoided with general potentials. Note that this classification does not specify how inflation ends, whether via a hybrid transition, or a violation of slow roll - the valley or the cliff.

Within this schema, we can consider $N$ parameter models. A Low- $\epsilon$ 1-Parameter model is almost entirely equivalent to standard $\Lambda \mathrm{CDM}$, since $\eta$ is the only nontrivial slow roll parameter. In this case the running in $\eta$ is effectively zero, which leads to a constant spectral index, with no tensor component. Conversely, a High- $\epsilon$ 1-Parameter model has no obvious analog, since $n_{s}, r, n_{t}$ and $\alpha$ are all specified in terms of a single parameter. Physically, setting $\eta=0$ means that $H(\phi)$ is a pure quadratic function. 
Since $\epsilon$ increases with time, a very small value of $\epsilon$ at CMB scales would imply that inflation must have ended via a sudden hybrid-style transition.

With two parameters, a High- $\epsilon$ model corresponds to $\Lambda \mathrm{CDM}+r$, where the tensor spectrum obviously has an inflationary prior that links the amplitude and spectral index. Moreover, as we will see later, the flow equations imply a weak scale dependence in $\epsilon$ and $\eta$. Thus, the running of the effective spectral index is non-zero - and for very high quality data, this increases the leverage we can obtain from slow roll reconstruction. Conversely, a Low- $\epsilon$, two parameter model corresponds to $\Lambda \mathrm{CDM}+\alpha$, but we now must be careful to ensure that for comparatively large and positive values of $\xi$ the total duration inflation is self-consistent. If we do wish to consider a large running at CMB scales, our one recourse is to add ${ }^{3} \lambda_{H}$ to our parameter set, leading to either a High- $\epsilon$ 4-Parameter model, or a Low- $\epsilon$ 3-Parameter model. In this case the tilt is transient, but we are left with a potential that has several free parameters. The classification scheme is summarized in Table 4 .

As we noted above, this analysis does not specify the mechanism that ends inflation - that is, whether we are rolling toward a cliff or a valley. However, since slow roll reconstruction effectively specifies the overall form of the potential, we can add further cuts to our parameter space by insisting on a sufficient overall duration of inflation, as described at the end of Section 2. This is not possible when using the spectral indices and amplitudes, since these variables contain no information about the duration of inflation.

\section{Error Estimation and Fisher Information}

For our error forecasts we make use of the Fisher information matrix [24], a measure of the width and shape of the likelihood function around its maximum,

$$
F_{i j}=-\left.\left\langle\frac{\partial^{2} L}{\partial \alpha_{i} \alpha_{j}}\right\rangle\right|_{\alpha=\bar{\alpha}},
$$

where $L \equiv \ln \mathcal{L}$ and the $\alpha_{i}$ denote model parameters. The Cramer-Rao inequality then says that the minimum possible standard deviation on a single parameter, $\alpha_{i}$, estimated

from the data is $1 / \sqrt{F_{i i}}$. This minimum standard deviation rises to $1 / \sqrt{\left(F^{-1}\right)_{i i}}$, if all parameters are estimated from the same data. Previous treatments of this topic include [67, 62], and we follow the formalism laid out in 62].

We restrict our attention to CMB data - in this paper, our principal concern is to compare slow roll reconstruction to fits to the spectral variables. Adding more information further constrains the free parameters, and thus accentuates the advantages the HSR formulation. Moreover, we are not considering the impact of priors based on the duration of inflation (which puts sharp cuts on the allowed region of parameter space), so what follows is essentially a worst case analysis for slow roll reconstruction.

Observations of the CMB measure the polarization and the anisotropy of the temperature of the radiation in terms of spherical harmonics, from which we obtain the $C_{\ell}$ for each of the spectra $C_{T \ell}, C_{E \ell}$, and $C_{B \ell}$ and the cross-correlation $C_{C \ell}$. Assuming 


\begin{tabular}{r|ccccc}
\hline \hline Experiment & $f_{\text {sky }}$ & $\begin{array}{c}\text { Frequency } \\
(\mathrm{GHz})\end{array}$ & $\begin{array}{c}\theta_{\text {beam }} \\
(')\end{array}$ & $\begin{array}{c}\sigma_{T} \\
(\mu K)\end{array}$ & $\begin{array}{c}\sigma_{P} \\
(\mu K)\end{array}$ \\
\hline Planck (Plk) & 0.65 & 143 & 8.0 & 5.2 & 10.8 \\
& & 217 & 5.5 & 11.7 & 24.3 \\
\hline Ideal Sat (Ideal). & 0.8 & $30-200$ & 8.0 & 2.2 & 2.2 \\
\hline \hline
\end{tabular}

Table 2. Experimental specifications for CMB satellites.

that the CMB multipoles are Gaussian distributed and letting $\alpha$ denote our vector of parameters, with $\bar{\alpha}$ the fiducial values, the Fisher matrix for a temperature/polarization measurement can be written

$$
F_{i j}=\left.\sum_{X, Y} \sum_{\ell} \frac{\partial C_{\ell}^{X}}{\partial \alpha_{i}}\left(\mathbf{C}_{\ell}^{X Y}\right)^{-1} \frac{\partial C_{\ell}^{Y}}{\partial \alpha_{j}}\right|_{\alpha=\bar{\alpha}} .
$$

We assume a gaussian beam profile and assume that foregrounds are perfectly subtracted. The elements of the symmetric matrix $\mathbf{C}_{\ell}^{X Y}$ are enumerated in [62]. We specify a proposed experiment in terms of $\sigma_{b}=\Theta_{\text {FWHM }} / \sqrt{8 \ln 2}$, the Gaussian beamwidth, where $\Theta_{\text {FwHM }}$ denotes the "full width at half maximum" power of the beam. The noise per multipole is $n_{0}=\sigma_{p i x}^{2} \Omega_{p i x}$, where $\Omega_{p i x}=\Theta_{\text {FWHM }}^{2}=4 \pi f_{s k y} / N_{p i x}$ is the beam solid angle, $N_{p i x}$ is the number of pixels (independent beams) in the survey region and $f_{s k y}$ is the fraction of the sky covered by observations [62]. The variance per pixel is $\sigma_{p i x}^{2}$ which can be obtained from the detector sensitivity as $\sigma_{p i x}=s / \sqrt{N t}$, where $N$ is the number of detectors and $t$ the integration time per pixel.

In this work we consider three cases:

(i) A cosmic variance limited survey $-n_{0}=0$, (C.V.),

(ii) The projected errors from the Planck satellite, (Plk),

(iii) The ideal satellite experiment of [62] (Ideal).

For all the above experiments we take $\ell_{\max }=1500$, unless stated otherwise. For the Planck satellite, we follow [27] and [67] and use only two of the ten available channels (143 and $217 \mathrm{GHz}$ ), assuming that the remaining channels have been used for foreground subtraction. This simple estimate leads to somewhat optimistic predictions for Planck's sensitivity to a primordial B-mode. However, since we are interested in the differences between the standard spectral analysis and slow roll reconstruction, it suffices for our needs. For the full sky "Ideal" experiments, we assume five identical channels at frequencies 30, 50, 70, 100 and $200 \mathrm{GHz}$. The satellite noise specifications are shown in Table 2, In all cases (except where explicitly specified) we include the effects of gravitational lensing of the $E$-mode polarization into $B$-mode polarization [31], which is computed within CAMB. We do not consider cases in which the $B$-mode has been delensed. We also tested our code by checking we could recover the results of [62] in the cases which overlap with our assumptions here. 


\section{Parameterizations}

The Fisher matrix formalism forecasts the likely error ellipse for any given fiducial model - and the size of this ellipse is a function of the chosen central parameter values. For concreteness, we assume that the non-inflationary parameters are well described by a $\Lambda \mathrm{CDM}$ cosmology. In what follows we set the central values of the baryon fraction, $\Omega_{b}$; the cold dark matter fraction, $\Omega_{c d m}$; the reduced Hubble parameter, $h$; and the optical depth to reionization, $\tau$; to their central values found in the WMAP3 concordance cosmology. As noted earlier, consistency with an inflationary prior requires $\Omega_{\text {Total }}=1$. Specifically we fix $\Omega_{\Lambda}=1-\Omega_{c d m}-\Omega_{b}$ and assume the dark energy has the equation of state $w=-1$. We ignore the neutrino mass.

We now compare models parameterized in terms of spectral variables, (the amplitude, $A\left(k_{0}\right)$; the scalar index, $n_{s}$; and the running, $\alpha$; as well as the corresponding tensor variables) to an HSR analysis. We define our amplitude variable $A\left(k_{0}\right)$ via

$$
P\left(k_{0}\right)=2.95 \times 10^{-9} A\left(k_{0}\right) .
$$

In the analysis below we always impose the simple inflationary prior on the tensor spectrum $n_{t}=-r / 8$, where $r$ is the ratio of the tensor and scalar amplitudes. This gives a considerable advantage to the spectral variables, since a generic treatment of the tensor modes would not assume this correlation, whereas it is implicit in the HSR formalism. However, it is simple to apply and used in most analyses of CMB data; so we adopt it here.

The likely parameter constraints on spectral variables are well understood (see 62 for instance), but this is the first forecast of the expected errors for HSR parameters. We will show that with very high quality data the HSR formalism results in a more sharply peaked likelihood surface, as it can make use of higher order correlations between slow roll variables. Since we can place cuts on the HSR parameter space using an "e-folds" prior, which is not included in our Fisher matrix analysis, the following treatment will necessarily underestimate the strength of the slow roll reconstruction formalism. Ironically, this is particularly noticeable with less precise data - if the likelihood contours are relatively large, they are more likely to extend into regions excluded by an e-folds prior.

\section{The Lever-arm effect}

It is intuitively clear that extending the range of wavenumbers (typically measured in terms of the number of decades of $k$ spanned by the data) over which we have CMB data will tighten the parameter bounds. However, if the spectral indices are very well approximated by the lowest order slow roll expressions $\left(n_{s}=1+2 \eta-4 \epsilon\right.$ and $\left.r=16 \epsilon\right)$, and $\epsilon$ and $\eta$ do not change significantly as observable modes leave the horizon, the slow roll formalism amounts to a linear transformation of the spectral variables. In terms of 
the spectral indices and running, the spectra take the form

$$
P_{\mathcal{R}}(k)=A_{s}^{2}\left(\frac{k}{k_{0}}\right)^{n_{s}(k)-1}
$$

where

$$
n_{s}(k)=n_{s}\left(k_{0}\right)+\frac{\alpha}{2} \ln \left(\frac{k}{k_{0}}\right)+\ldots,
$$

and

$$
P_{h}(k)=A_{s}^{2} r\left(\frac{k}{k_{0}}\right)^{n_{t}},
$$

where $n_{t}$ is assumed to be scale invariant.

The extra information exploited by slow roll reconstruction comes from the hierarchy of consistency relations that exist between the spectral parameters at all orders in slow roll. Consider, for example, the case in which we have two spectral parameters, $\left\{n_{s}, r\right\}$, which is analogous to the High- $\epsilon$, 2-Parameter inflationary model, specified via $\{\epsilon, \eta\}$. In the spectral parametrization, $\alpha=0$, but slow roll requires

$$
\alpha \approx-\frac{1}{1-\epsilon}\left[8 \epsilon^{2}-10 \epsilon \eta\right] \approx-\epsilon[8 \epsilon-10 \eta](1+\epsilon) .
$$

The corresponding spectral model $\left\{n_{s}, r\right\}$ has only the term linear in $\ln \left(k / k_{0}\right)$ in $\ln \left(P_{\mathcal{R}}(k)\right)$, whereas the slow roll parametrization has additional terms of the form $\mathcal{O}(\epsilon, \eta, \ldots) \ln \left(k / k_{0}\right)^{2}$. Even though the co-efficient is small, when the lever arm (in $\left.\ln k / k_{0}\right)$ is large the quadratic terms will make a substantial contribution to the spectrum.

One can show that, to a good approximation, derivatives of the $C_{\ell}$ with respect to the parameters that specify the power spectrum can be written as

$$
\left.\frac{1}{C_{\ell}} \frac{\partial C_{\ell}}{\partial \alpha_{i}} \approx \frac{1}{P(k)} \frac{\partial P(k)}{\partial \alpha_{i}}\right|_{k=\ell /\left(\eta_{0}-\eta_{*}\right)},
$$

where $\alpha_{i}=n_{s}, d n_{n} / d \ln k, \epsilon, \eta$ etc and $P(k)$ is the appropriate spectrum (scalar for temperature and $E$-mode, tensor for $B$-mode). Here the quantity $\eta_{0}-\eta_{*}$ denotes the conformal time interval between today $\left(\eta_{0}\right)$ and last scattering $\left(\eta_{*}\right)$ and is not to be confused with the slow roll parameter $\eta$. The elements of $F_{i j}$ for which $\alpha_{i}$ and $\alpha_{j}$ are both spectral parameters are approximately

$$
F_{i j}=\left.\sum_{\ell=2}^{\ell_{\max }} \frac{(2 \ell+1)}{2} \frac{\partial \ln P_{\mathcal{R}}(k)}{\partial \alpha_{i}} \frac{\partial \ln P_{\mathcal{R}}(k)}{\partial \alpha_{j}}\right|_{k=\ell /\left(\eta_{0}-\eta_{*}\right)} .
$$

If we are working with $\left\{n_{s}, \alpha, r\right\}$ we have

$$
\begin{aligned}
& \frac{\partial \ln C_{\ell}^{T}}{\partial n_{s}} \approx \frac{\partial \ln P_{\mathcal{R}}(k)}{\partial n_{s}}=\ln \left(\frac{k}{k_{0}}\right), \\
& \frac{\partial \ln C_{\ell}^{T}}{\partial \alpha} \approx \frac{\partial \ln P_{\mathcal{R}}(k)}{\partial \alpha}=\frac{1}{2} \ln \left(\frac{k}{k_{0}}\right)^{2}, \\
& \frac{\partial \ln C_{\ell}^{T}}{\partial r} \approx 0,
\end{aligned}
$$


where the last line signifies we are neglecting the contribution of tensor modes to the temperature anisotropies. Assuming the consistency relation,

$$
\begin{aligned}
& \frac{\partial \ln C_{\ell}^{B}}{\partial n_{s}} \approx \frac{\partial \ln P_{h}(k)}{\partial n_{s}}=0, \\
& \frac{\partial \ln C_{\ell}^{B}}{\partial \alpha} \approx \frac{\partial \ln P_{h}(k)}{\partial \alpha}=0, \\
& \frac{\partial \ln C_{\ell}^{B}}{\partial r} \approx \frac{\partial \ln P_{h}(k)}{\partial r}=1-\frac{1}{8} \ln \left(\frac{k}{k_{0}}\right) .
\end{aligned}
$$

The corresponding slow roll parameter set is $\{\epsilon, \eta, \xi\}$. Keeping the leading terms in slow roll multiplying each power of $\ln k / k_{0}$ we find

$$
\begin{aligned}
\frac{\partial \ln C_{\ell}}{\partial \epsilon} \approx & \frac{\partial \ln P_{\mathcal{R}}(k)}{\partial \epsilon}=\left(-4-4(1+\mathcal{C}) \epsilon-\frac{1}{2}(3-5 \mathcal{C}) \eta\right) \ln \left(\frac{k}{k_{0}}\right) \\
& +\frac{1}{2 !}\left[10 \eta-16 \epsilon+\frac{1}{2}(5-7 \mathcal{C}) \xi\right] \ln \left(\frac{k}{k_{0}}\right)^{2}-\frac{14}{3 !} \xi \ln \left(\frac{k}{k_{0}}\right)^{3},(4) \\
\frac{\partial \ln C_{\ell}}{\partial \eta} \approx & \frac{\partial \ln P_{\mathcal{R}}(k)}{\partial \eta}=\left(2-\frac{1}{2}(3-5 \mathcal{C}) \epsilon\right) \ln \left(\frac{k}{k_{0}}\right) \\
& +\frac{1}{2 !}\left(10 \epsilon-\frac{1}{2}(3-\mathcal{C}) \xi\right) \ln \left(\frac{k}{k_{0}}\right)^{2}+\frac{2}{3 !} \xi \ln \left(\frac{k}{k_{0}}\right)^{3} \\
\frac{\partial \ln C_{\ell}}{\partial \xi} \approx & \frac{\partial \ln P_{\mathcal{R}}(k)}{\partial \xi}=\frac{1}{2}(3-\mathcal{C}) \ln \left(\frac{k}{k_{0}}\right)-\frac{1}{2 !}\left(2-\frac{1}{2}(5-7 \mathcal{C}) \epsilon\right. \\
& \left.+\frac{1}{2}(3-\mathcal{C}) \eta\right) \ln \left(\frac{k}{k_{0}}\right)^{2}+\frac{1}{3 !}(2 \eta-14 \epsilon+(3-\mathcal{C}) \xi) \ln \left(\frac{k}{k_{0}}\right)^{3}(50)
\end{aligned}
$$

where in all instances, $k$ is understood to be $k=\ell /\left(\eta_{0}-\eta_{*}\right)$. Similar expressions exist for the tensor spectrum. As noted above, for a given number of parameters, the slow roll parametrization adds an extra power of $\ln \left(k / k_{0}\right)$, which is an expression of a higher order consistency condition [37, 38, 14]. This extra power provides more leverage on the likelihood space as one adds information further from the fiducial scale.

To compare the constraining power of the two parameterizations, we consider the volume of parameter space contained within the Fisher ellipses, as a function of $\ell_{\max }$, in analogy to the metric developed by the Dark Energy Task Force 68. For three slow roll or spectral parameters, this is the volume of an ellipsoid. In order to make a comparison between the two different parameterizations, we normalize the volume by its value when $\ell_{\max }$ is set to the pivot scale which, for $k_{0}=0.05 \mathrm{Mpc}^{-1}$, is $\ell \sim 500$. The constraining power of the parametrization determined by how quickly the volume contracts as information is added, and we plot a Figure of Merit, defined as the inverse of the normalized $n$-dimensional volume enclosed within the error ellipsoids. In reality, the CMB probes a relatively small range of scales (the largest value of $\ln \left(k / k_{0}\right)$ is likely on the order of 6 ), which limits the maximal extent of our lever arm. Moreover, the highest order term in $\ln \left(k / k_{0}\right)$ in the derivatives of the $C_{l}$ is multiplied by a slow roll parameter, which is necessarily considerably smaller than unity. Consequently, if we restrict attention solely to CMB data, we will need a very accurate measurement of 


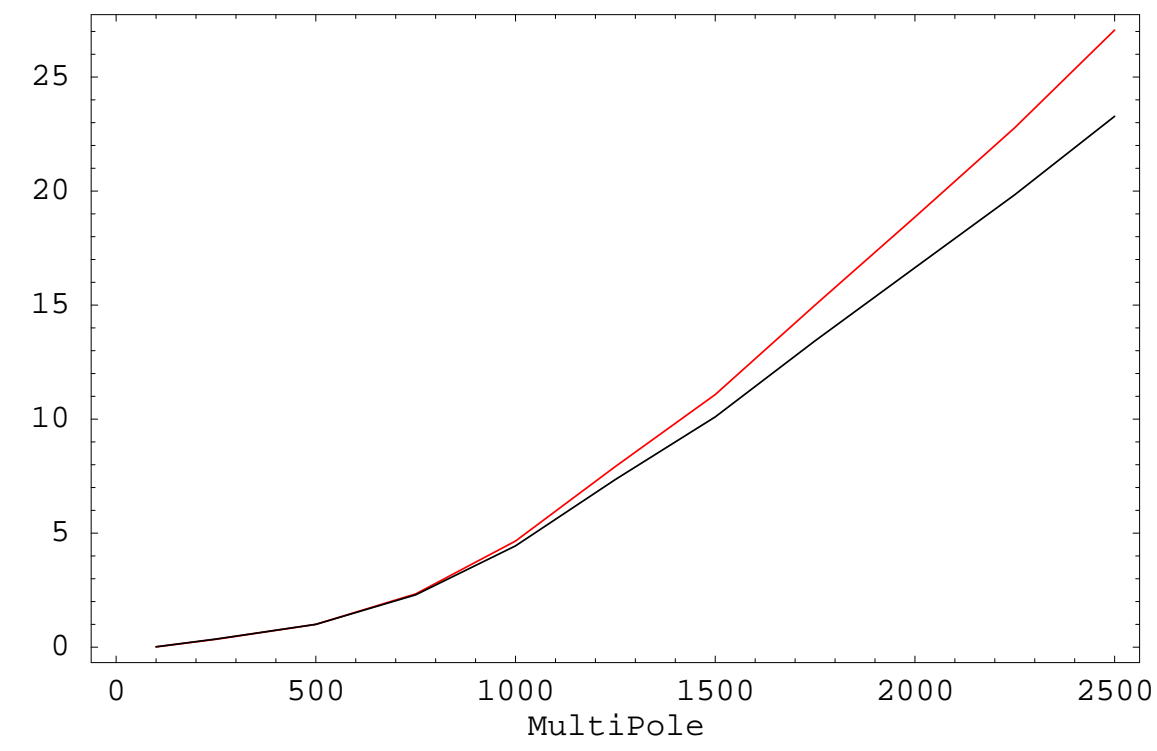

Figure 3. Model with $\epsilon=0.015, \eta=-0.02, \xi=0.0$. The figures of merit for the $\{\epsilon, \eta, \xi\}$ (red) and $\left\{r, n_{s}, \alpha\right\}$ parameterizations (black), normalized at $\ell=500$, calculated using the full Fisher matrix with cosmic variance only. As the lever arm in $\ell$ becomes longer, the extra information in the slow roll formalism leads to a more sharply peaked likelihood function and thus tighter constraints on the model parameters.

the primordial CMB to make use of the extra information. To examine our hypothesis, however, we can look at extreme cases. From equation (39), the largest deviations are found in models where $\epsilon$ is large, and $\epsilon$ and $\eta$ have opposite signs. For example, consider $\epsilon=0.015$ and $\eta=-0.02$, corresponding to spectral parameters $r=0.23, n_{s}=0.9$ and $\alpha=0.0$. Figure 3 shows the Figures of Merit for the spectral and slow roll formulations for this parameter set, and the two values have begun to diverge once $\ell_{\max }>1500$. This is a gedanken experiment, given both that we assumed foreground subtraction and that $n_{s}-1$ and $r$ are unrealistically large. This illustrates that second order consistency conditions are probably not testable by CMB data alone. However, it also gives cause for optimism that this lever arm effect can be exploited by combinations of CMB surveys and datasets sensitive to the primordial spectrum at shorter scales.

Adding further decades in information from the power spectrum will increase the difference between the figures or merit. However, we also see that the running induced solely by $\epsilon$ and $\eta$ being non-zero is proportional to $\epsilon$. Thus in Low- $\epsilon$ models (corresponding to those with a negligible value of $r$ ), increasing the lever arm in $\ln k / k_{0}$ will not give any extra advantage to slow roll reconstruction, relative to the spectral variables, unless we are lucky enough to see scale dependence $\xi$ induced by a non-zero $\eta$. Moreover, if we do observe a running in conjunction with a low value of $r$, then we will obtain an unambiguous measurement of $\xi$. 


\begin{tabular}{|c|l|llllll|}
\hline Parameter & Fiducial & C.V. Abs & C.V. \% & Ideal Abs. & Ideal \% & Plk Abs & Plk \% \\
\hline$\omega_{b}$ & 0.024 & 0.0000607 & 0.253 & 0.0000772 & 0.322 & 0.00018 & 0.75 \\
$\omega_{c d m}$ & 0.12 & 0.000293 & 0.244 & 0.000503 & 0.419 & 0.0014 & 1.17 \\
$H_{0}$ & 72.0 & 0.132 & 0.184 & 0.225 & 0.312 & 0.664 & 0.922 \\
$\tau$ & 0.164 & 0.0026 & 1.59 & 0.00306 & 1.87 & 0.000637 & 3.88 \\
$A\left(k_{0}\right)$ & 0.9 & 0.00437 & 0.486 & 0.00547 & 0.607 & 0.0116 & 1.19 \\
\hline \hline$\epsilon$ & 0.01 & 0.000122 & 1.22 & 0.00021 & 2.1 & 0.00181 & 18.1 \\
$\eta$ & 0.0 & 0.00151 & - & 0.00206 & - & 0.00331 & - \\
$\xi$ & 0.0 & 0.00174 & - & 0.00216 & - & 0.00285 & - \\
\hline \hline$\epsilon$ & 0.01 & 0.000122 & 1.22 & 0.000209 & 2.09 & 0.00179 & 17.9 \\
$\eta$ & 0.0 & 0.000871 & - & 0.00115 & - & 0.00417 & - \\
\hline \hline$\epsilon$ & 0.01 & 0.000116 & 1.16 & 0.000193 & 1.93 & 0.000914 & 9.14 \\
\hline
\end{tabular}

Table 3. Errors for model: $\epsilon=0.01, \eta=0.0, \xi=0.0$. This model gives 49.5 e-folds of inflation after the fiducial scale leaves the horizon. Here C.V. refers to a cosmic variance limited survey, Ideal is the "straw man" satellite proposal of 62 and Plk refers to Planck (although this analysis assumes perfect foreground subtraction, and thus over-estimates Planck's capabilities, especially at small $r$ ). We give absolute and percentage errors for each parameter. In all cases we assume $\ell_{\max }=1500$. The second block gives the forecast for a fit to $\{\epsilon, \eta, \xi\}$, the middle block for a fit to $\{\epsilon, \eta\}$ and the bottom block give the results for a fit to $\epsilon$ alone. As expected the constraints get tighter as the number of parameters is reduced. Finally the top block gives the forecasts for the other cosmological parameters, derived from the three parameter slow roll fit.

\section{Forecasts}

We use the derivative methodology outlined in [27] to compute the numerical derivatives using CAMB [26]. We consider the parameters 'class-by-class' as described in \$4, beginning with the simplest possible case, the High- $\epsilon$ 1-Parameter model. In each case where one or more of the parameters is zero, we consider both situations in which we fit for the parameter at its zero value and the alternative in which it is not included in the parameter set. This second case reduces the dimensionality of the parameter space by one and naturally leads to tighter restrictions on the other parameters. For all but the High- $\epsilon$ 1-Parameter models, we work with a fiducial point chosen so that $n_{s}=0.97$ at the pivot. That is, $r$ sets $\epsilon$ while $\alpha$ sets $\xi$ and then both $\epsilon$ and $\xi$ are used to set $\eta$ keeping $n_{s}=0.97$.

High-e 1-Parameter Models: This is perhaps the simplest possible model of inflation and effectively describes an $m^{2} \phi^{2}$ potential. However, the actual portion of the potential sampled by inflation is not specified, and if this turns out to be far from the minimum inflation must end via a hybrid transition. We consider $\epsilon=0.01$ and $\epsilon=0.0025$. Via equation A.2 the first case has a further 49.5 e-folds of inflation after the fiducial scale has left the horizon and gives a tilt of $n_{s}=0.96$. The second case has 199.5 further 


\begin{tabular}{|c|l|llllll|}
\hline Parameter & Fiducial & C.V. Abs & C.V. \% & Ideal Abs. & Ideal \% & Plk Abs & Plk \% \\
\hline$\epsilon$ & 0.0025 & 0.0000522 & 2.09 & 0.000112 & 4.48 & 0.000826 & 33. \\
$\eta$ & 0.0 & 0.00151 & - & 0.00206 & - & 0.00369 & - \\
$\xi$ & 0.0 & 0.00172 & - & 0.00214 & - & 0.00332 & - \\
\hline \hline$\epsilon$ & 0.0025 & 0.0000522 & 2.09 & 0.000112 & 4.48 & 0.000825 & 33 \\
$\eta$ & 0.0 & 0.00083 & - & 0.00108 & - & 0.00266 & - \\
\hline \hline$\epsilon$ & 0.0025 & 0.0000517 & 2.07 & 0.000109 & 4.38 & 0.000665 & 26.6 \\
\hline
\end{tabular}

Table 4. Errors for model: $\epsilon=0.0025, \eta=0.0, \xi=0.0$. This model gives rise to 199.5 e-folds of inflation after the fiducial scale leaves the horizon, and we have used the same conventions as Table 3

e-folds and a tilt of $n_{s}=0.99$. This model has no obvious analogue in spectral variables, so we only present forecasts for the slow roll variables. As can be seen from Tables 3 and 4, this one parameter model can be very tightly constrained. However, the precise measurement of $r$ is facilitated by the correlation of $r$ and $n_{s}$, and the latter is very tightly constrained by measurements of the temperature anisotropies.

Low- $\epsilon$ 1-Parameter Models: In this case, the tensor signal is tiny, and $|\eta| \neq 0$. Table 5 shows the forecast errors for a model $\eta$ so that $n_{s}=0.97$ and $\alpha=0$, so $\eta=-0.015$ and $\xi=0.0$. This scenario model leads to $\sim 600$ e-folds of inflation, so we would clearly require a hybrid transition. Note that when $\xi$ and $\alpha$ are marginalized over, the relative constraints on $n_{s}-1$ and $\eta$ are identical. We make no forecast for $\epsilon$ - this fit is analogous to that of a pure $\Lambda \mathrm{CDM}$ cosmology. This forecast includes the $B$-mode contribution from the lensed $E$-mode. We present the same forecast in Table 6, with the $B$-mode spectrum omitted from our analysis - that is all derivatives of $C^{B B}$ are assumed to vanish. We see that in the absence of primordial tensors, a highly accurate $B$-mode measurement would significantly improve constraints on $\omega_{c d m}$, since the lensing is induced by the dark matter potential wells - although whether this level of accuracy is achievable in practice is of course a very different matter.

We give forecasts for both slow roll and spectral variables. We construct the analogous spectral variables by computing them at the pivot. The resulting spectra are subtly different, since a two parameter slow roll model has a non-zero running if $\epsilon$ is not minuscule; this effect will necessarily be absent in the spectral case. If we assume that the underlying cosmology is a Low- $\epsilon$ 1-Parameter model, we are still free to include $\xi$ and $\alpha$ in our parameter set. In this case, $\alpha \approx-2 \xi$ and we see that the constraints on $\xi$ indeed match those on $\alpha$. However, adding $\xi$ weakens the constrain on $\eta$ since $n_{s}$ has a partial degeneracy between $\alpha$ and $\eta$. This amounts to a rotation in our parameter space, and does not imply that slow roll constraints are intrinsically less accurate than the spectral constraints. We implement "Low- $\epsilon$ " scenarios by using the full slow-roll hierarchy with $\epsilon$ set to a very low value $\left(10^{-10}\right)$. The results are insensitive to the actual value of $\epsilon$, provided it is far below the threshold of detectability. 


\begin{tabular}{|c|l|llllll|}
\hline Parameter & Fiducial & C.V. Abs & C.V. \% & Ideal Abs. & Ideal \% & Plk Abs & Plk \% \\
\hline$\omega_{b}$ & 0.024 & 0.0000614 & 0.256 & 0.0000778 & 0.324 & 0.000182 & 0.758 \\
$\omega_{c d m}$ & 0.12 & 0.000311 & 0.259 & 0.00051 & 0.425 & 0.00142 & 1.19 \\
$H_{0}$ & 72. & 0.141 & 0.195 & 0.229 & 0.317 & 0.676 & 0.939 \\
$\tau$ & 0.164 & 0.00281 & 1.71 & 0.00328 & 2. & 0.00647 & 3.94 \\
$A\left(k_{0}\right)$ & 0.9 & 0.00468 & 0.52 & 0.00577 & 0.641 & 0.0117 & 1.3 \\
\hline \hline$n_{s}-1$ & -0.03 & 0.00166 & 5.53 & 0.00215 & 7.16 & 0.00439 & 14.3 \\
$\alpha$ & 0. & 0.00344 & - & 0.00428 & - & 0.0066 & - \\
\hline \hline$n_{s}-1$ & -0.03 & 0.00166 & 5.53 & 0.00212 & 7.07 & 0.00427 & 14.2 \\
\hline \hline$\eta$ & -0.015 & 0.00308 & 20.6 & 0.00404 & 26.9 & 0.00632 & 42.1 \\
$\xi$ & 0. & 0.00346 & - & 0.00432 & - & 0.00669 & - \\
\hline \hline$\eta$ & -0.015 & 0.000828 & 5.52 & 0.00106 & 7.05 & 0.00213 & 14.2 \\
\hline
\end{tabular}

Table 5. Errors for model $\epsilon=10^{-10}, \eta=-0.015$ and $\xi=0.0$, where the CMB spectra include the $B$-mode contribute from the lensed $E$ mode. The conventions here match those of Table 3, although we also give forecasts for the corresponding spectral parameters.

\begin{tabular}{|c|l|llllll|}
\hline Parameter & Fiducial & C.V. Abs & C.V. \% & Ideal Abs. & Ideal \% & Plk Abs & Plk \% \\
\hline$\omega_{b}$ & 0.024 & 0.000066 & 0.275 & 0.0000831 & 0.346 & 0.000192 & 0.801 \\
$\omega_{c d m}$ & 0.12 & 0.000634 & 0.528 & 0.000755 & 0.63 & 0.00155 & 1.29 \\
$H_{0}$ & 72. & 0.271 & 0.376 & 0.33 & 0.459 & 0.741 & 1.03 \\
$\tau$ & 0.164 & 0.00297 & 1.81 & 0.00338 & 2.06 & 0.00642 & 3.92 \\
$A\left(k_{0}\right)$ & 0.9 & 0.00574 & 0.638 & 0.00657 & 0.73 & 0.00117 & 1.3 \\
\hline$n_{s}-1$ & -0.03 & 0.00204 & 6.8 & 0.00248 & 8.23 & 0.00459 & 15.3 \\
$\alpha$ & 0. & 0.00377 & - & 0.00443 & - & 0.00653 & - \\
\hline \hline$n_{s}-1$ & -0.03 & 0.00198 & 6.6 & 0.00237 & 7.9 & 0.00457 & 15.2 \\
\hline \hline$\eta$ & -0.015 & 0.00363 & 24.2 & 0.00436 & 29.1 & 0.00632 & 42.1 \\
$\xi$ & 0. & 0.0038 & - & 0.00448 & - & 0.00662 & - \\
\hline \hline$\eta$ & -0.015 & 0.00099 & 6.6 & 0.00119 & 7.9 & 0.00228 & 15.2 \\
\hline
\end{tabular}

Table 6. Errors for model $\epsilon=10^{-10}, \eta=-0.015$ and $\xi=0.0$. In this case we did not include any $B$-mode information in our forecasts. The forecasts for our measurement of $\omega_{c d m}$ are considerably looser than those found in Table 5 even though the primordial $B$ mode is entirely absent, since the amplitude of the $B$ mode is now entirely dependent on the depth of the dark matter potential wells, and we are not exploiting this information here. 


\begin{tabular}{|c|l|llllll|}
\hline Parameter & Fiducial & C.V. Abs & C.V. \% & Ideal Abs. & Ideal \% & Plk Abs & Plk \% \\
\hline$\omega_{b}$ & 0.024 & 0.0000607 & 0.254 & 0.0000772 & 0.322 & 0.00018 & 0.75 \\
$\omega_{c d m}$ & 0.12 & 0.000305 & 0.254 & 0.000516 & 0.43 & 0.00141 & 1.18 \\
$H_{0}$ & 72.0 & 0.136 & 0.189 & 0.23 & 0.319 & 0.67 & 0.93 \\
$\tau$ & 0.164 & 0.00271 & 1.66 & 0.00318 & 1.94 & 0.0064 & 3.9 \\
$A\left(k_{0}\right)$ & 0.9 & 0.00451 & 0.501 & 0.00558 & 0.62 & 0.0116 & 1.29 \\
\hline \hline$r$ & 0.24 & 0.0026 & 1.08 & 0.00425 & 1.77 & 0.351 & 14.6 \\
$n_{s}-1$ & -0.03 & 0.00167 & 5.57 & 0.00217 & 7.23 & 0.00427 & 14.2 \\
$\alpha$ & 0.0 & 0.00355 & - & 0.0044 & - & 0.00675 & - \\
\hline \hline$r$ & 0.24 & 0.0026 & 1.08 & 0.00425 & 1.77 & 0.0348 & 14.5 \\
$n_{s}-1$ & -0.03 & 0.00167 & 5.57 & 0.00213 & 7.1 & 0.00425 & 14.2 \\
\hline \hline$\epsilon$ & 0.015 & 0.000158 & 1.06 & 0.000259 & 1.73 & 0.00213 & 14.3 \\
$\eta$ & 0.015 & 0.00154 & 10.3 & 0.00209 & 13.9 & 0.00425 & 34.1 \\
$\xi$ & 0.0 & 0.00176 & - & 0.00218 & - & 0.00337 & - \\
\hline \hline$\epsilon$ & 0.015 & 0.000157 & 1.05 & 0.000257 & 1.72 & 0.00269 & 13.9 \\
$\eta$ & 0.015 & 0.000911 & 6.08 & 0.00122 & 8.11 & 0.00483 & 32.2 \\
\hline
\end{tabular}

Table 7. Errors for model: $\epsilon=0.015, \eta=0.015, \xi=0.0$

High- $\epsilon$ 2-Parameter Models: If we consider only scalar perturbations High- $\epsilon$ 2Parameter models have a degeneracy in the $\epsilon-\eta$ plane, which is broken by the limits on $\epsilon$ derived from $B$-mode observations, and/or the running of the spectral index. As we showed in Figure 3, the constraints on the slow roll parameters will tighten more rapidly than the analogous ones on the spectral parameters when $\epsilon$ is relatively large thanks to the lever arm effect. In the forecast presented in Table 7 we have set $\epsilon=0.015$ and chosen $\eta$ so that $n_{s}=0.97$. However, since our forecasts have been done with $\ell_{\max }=1500$ we do not see the benefit of the lever arm effect here.

Low- $\epsilon$ 2-Parameter Models: We now consider the scenario in which the inflaton potential is specified by $\eta$ and $\xi$. That is, $r=0, n_{s} \neq 1$ and $\alpha \neq 0$. In this case, the slow roll parametrization is (almost) identical to the spectral parametrization. There will be terms proportional to $\xi \ln \left(k / k_{0}\right)^{3}$, but these are never large over the range of scales probed by the CMB. In computing the errors for these models, we do not include information from lensing of the $E$-mode polarization into $B$-mode polarization, so we have somewhat over-estimated the likely errors for $\omega_{c d m}$. However, both the spectral and slow roll variables will be equally affected.

The parameter $\xi$ dominates the dynamics very quickly when $\xi>0$ [69], and thus $\alpha<0$. In Appendix A we present an analytic treatment of the slow roll dynamics for the Low- $\epsilon$ 2-Parameter model, from which we can compute the remaining number of e-folds. Fixing $n_{s}=0.97$ and then decreasing $\alpha$ from zero leads to a dramatic decrease in $N_{e}$, as shown in Figure A1, For $\eta=-0.015, \xi=0.0$ inflation ends after $\sim 600$ e-folds, 


\begin{tabular}{|c|l|llllll|}
\hline Parameter & Fiducial & C.V. Abs & C.V. \% & Ideal Abs. & Ideal \% & Plk Abs & Plk \% \\
\hline$n_{s}-1$ & -0.03 & 0.002 & 6.67 & 0.00247 & 8.23 & 0.00459 & 15.3 \\
$\alpha$ & -0.004 & 0.00378 & 94.4 & 0.0044 & 111. & 0.00653 & 163. \\
\hline \hline$\eta$ & -0.0165 & 0.0019 & 11.6 & 0.00228 & 13.8 & 0.00343 & 20.9 \\
$\xi$ & 0.002 & 0.0019 & 97.1 & 0.00225 & 112. & 0.00331 & 165. \\
\hline
\end{tabular}

Table 8. Errors for model: $\epsilon=10^{-10}, \eta=-0.0165, \xi=0.002$

\begin{tabular}{|c|l|llllll|}
\hline Parameter & Fiducial & C.V. Abs & C.V. \% & Ideal Abs. & Ideal \% & Plk Abs & Plk \% \\
\hline$n_{s}-1$ & -0.03 & 0.002 & 6.67 & 0.00236 & 7.87 & 0.00459 & 15.3 \\
$\alpha$ & -0.01 & 0.00378 & 37.8 & 0.00444 & 44.4 & 0.00655 & 65.5 \\
\hline \hline$\eta$ & -0.019 & 0.0019 & 10.2 & 0.00228 & 12.2 & 0.00344 & 18.5 \\
$\xi$ & 0.005 & 0.00195 & 39 & 0.00225 & 45 & 0.00332 & 66.4 \\
\hline
\end{tabular}

Table 9. Errors for model: $\epsilon=10^{-10}, \eta=-0.019, \xi=0.005$

\begin{tabular}{|c|l|llllll|}
\hline Parameter & Fiducial & C.V. Abs & C.V. \% & Ideal Abs. & Ideal \% & Plk Abs & Plk \% \\
\hline$n_{s}-1$ & -0.03 & 0.002 & 6.67 & 0.00244 & 8.13 & 0.00457 & 15.2 \\
\hline$\alpha$ & -0.06 & 0.00381 & 6.36 & 0.00449 & 7.48 & 0.00672 & 11.2 \\
\hline \hline$\eta$ & -0.037 & 0.00182 & 4.91 & 0.00222 & 6.02 & 0.00335 & 9.09 \\
\hline$\xi$ & 0.03 & 0.00189 & 6.31 & 0.00223 & 7.44 & 0.00333 & 11.1 \\
\hline
\end{tabular}

Table 10. Errors for model: $\epsilon=10^{-10}, \eta=-0.037, \xi=0.03$

while $\xi=10^{-5}$ gives $\sim 260$ e-folds and $\xi=0.001$ yields only $\sim 55$ efolds, even though the corresponding value of $\alpha$ at the pivot is very small. At this point we are beginning to bite into the e-foldings window of equation (25). Almost any prior on the number of e-folds will lead to tight constraints on $\xi$ [8]. This constraint has has no analog when the power spectrum is specified in terms of $n_{s}$, and $\alpha$, and allows slow roll reconstruction to impose much tighter constraints than fits to $n_{s}$, and $\alpha$.

For this set of parameters, we find that the smallest detectable value of $\xi$ (assuming a cosmic variance limited measurement of the CMB) is $\xi \sim 0.002$. Consequently, if $\xi$ can be detected in via fits to CMB data, the end of inflation was 'in sight' as the fiducial modes left the horizon, and we do not need to posit a hybrid transition. Given the difficulties of foreground subtraction, a constraint this tight would almost certainly require a combined fit to several orthogonal datasets. Furthermore, as pointed out by [70], the extra parameters we have ignored (neutrino mass, dark matter equation of state), can have a non-negligible effect on estimates of primordial spectrum parameters and thus forecasts. We demonstrate our ability to constrain $\xi$ in Tables 8-10. In each case we forecast the errors with $\eta$ and $\xi$ chosen so that $n_{s}=0.97$, and with increasing values of $\xi$. 


\begin{tabular}{|c|l|llllll|}
\hline Parameter & Fiducial & C.V. Abs & C.V. \% & Ideal Abs. & Ideal \% & Plk Abs & Plk \% \\
\hline$r$ & 0.24 & 0.0026 & 1.08 & 0.00424 & 1.77 & 0.0351 & 14.6 \\
\hline$n_{s}-1$ & -0.03 & 0.00167 & 5.57 & 0.00216 & 7.2 & 0.00427 & 14.2 \\
\hline$\alpha$ & -0.002 & 0.00349 & 175 & 0.00436 & 218 & 0.00669 & 335 \\
\hline \hline$\epsilon$ & 0.015 & 0.000158 & 1.05 & 0.000258 & 1.72 & 0.00196 & 13.1 \\
\hline$\eta$ & 0.14 & 0.00154 & 11.0 & 0.00209 & 14.9 & 0.00489 & 34.9 \\
\hline$\xi$ & 0.001 & 0.00176 & 176 & 0.00218 & 218 & 0.00336 & 336 \\
\hline
\end{tabular}

Table 11. Errors for model: $\epsilon=0.015, \eta=0.014, \xi=0.001$. This model gives $\sim 33$ e-foldings of inflation after the fiducial scale leaves the horizon.

\begin{tabular}{|c|l|llllll|}
\hline Parameter & Fiducial & C.V. Abs & C.V. \% & Ideal Abs. & Ideal \% & Plk Abs & Plk \% \\
\hline$r$ & 0.24 & 0.00257 & 1.08 & 0.00422 & 1.78 & 0.0348 & 14.6 \\
\hline$n_{s}-1$ & -0.03 & 0.00167 & 5.57 & 0.00216 & 7.2 & 0.00427 & 14.2 \\
\hline$\alpha$ & -0.02 & 0.00357 & 17.9 & 0.00442 & 22.1 & 0.00683 & 34.2 \\
\hline \hline$\epsilon$ & 0.015 & 0.000158 & 1.06 & 0.00026 & 1.73 & 0.00213 & 14.2 \\
\hline$\eta$ & 0.0075 & 0.00152 & 20.4 & 0.00207 & 27.8 & 0.00509 & 68.3 \\
\hline$\xi$ & 0.01 & 0.00176 & 17.6 & 0.00218 & 21.8 & 0.00337 & 33.7 \\
\hline
\end{tabular}

Table 12. Errors for model: $\epsilon=0.015, \eta=0.0075, \xi=0.01$. This model gives only $\sim 12$ e-foldings of inflation after the fiducial scale leaves the horizon.

High-e 3-Parameter Models: Finally, we consider the case in which the first three slow roll parameters are non-zero. In this case $\epsilon$ can be large, and the slow roll variables can again evolve within the observable window. We present results for two representative cases in Tables 11 and 12. However, the principal constraining power of slow roll reconstruction, relative to the usual spectral formulation, is again its ability to include restrictions based on the duration of inflation. For example, the model in Table 12 would manage a bare 12 e-folds of inflation. If we found these central values for $\{\epsilon, \eta, \xi\}$, we would know this description of the inflaton potential was either incomplete or that the primordial spectrum was not generated by slow roll inflation.

\section{Conclusions}

We have considered the constraints one can place on the inflationary parameter space via slow roll reconstruction. In the course of this work we also analyzed the precision of different approximation schemes that can be used to compute the underlying spectrum. As noted in [7], the primordial spectrum is easily computed by numerically solving the mode equations (13,14). In this case slow roll reconstruction contains no approximations, as the slow roll hierarchy itself is solved analytically [16]. For almost all models of interest, approximate solutions will be more than adequate given the precision of current data. The principal concern is that different schemes for computing the spectrum will impose implicit priors on the duration of inflation, and these will effect 
the computed likelihood contours if they are not properly understood. This issue was investigated in Refs. [17, 18, which implement slow roll reconstruction with an exact solver for the perturbation spectrum; they find that the sole difference in likelihood contours using current data can be attributed to implicit priors on the duration of inflation. However, since the spectrum can be computed numerically at little cost, using the exact solver has the advantage of removing a possible source of uncertainty.

In addition, we introduce a scheme for classifying inflationary models, based on the order at which the slow roll hierarchy is truncated, and whether the $\epsilon$ parameter is small enough for its contribution to the inflationary dynamics to be ignored. We refer to these models as "Low- $\epsilon$, N-parameter" and "High- $\epsilon$, N-parameter" models. In the former case, we can impose the slow roll prior while ignoring a possible tensor contribution to the $\mathrm{CMB}$, in analogy with the usual $\Lambda$ CDM parameter set of the concordance cosmology. Physically, $\epsilon$ responds to the ratio of the amplitude of tensor and scalar perturbations, which reflects the energy scale at which inflation occurs. It is possible (and some might say likely, if one expects inflation to be consistent with the microphysical constraints of string theory) the inflationary scale is well below the GUT scale. In this case $\epsilon$ and the amplitude of the tensor spectrum must be vanishingly small. In this limit $\epsilon$ effectively decouples from the slow roll hierarchy.

Slow roll reconstruction can lead to tighter constraints on the parameter space than those found with the usual spectral indices and runnings. In particular, slow roll reconstruction allows us to impose constraints on the total duration of inflation, because slow roll reconstruction requires one to compute the duration of the inflationary era for a given set of parameters. If this value is very low - as it can be for apparently reasonable parameters - we do not have a self-consistent description of inflation. This effect is clearly seen in implementations of slow roll reconstruction [6, 7]. Constraints based on the duration of inflation imply a sharp cut on the relevant parameter space, and this effect is not captured by our Fisher matrix forecasts which assume a Gaussian likelihood. These constraints simply encode our assumption that we are using enough slow-roll parameters to provide a full description of the inflationary potential, and do not introduce any significant new or ad hoc assumptions into the parameter estimates. The impact of this constraint could be forecast by running Markov Chains on simulated data for a proposed experiment. We sketch an improved " $\epsilon$-dependent" algorithm for imposing the "e-fold prior" that will be explored in detail in future work.

Slow roll reconstruction can also lead to improved constraints via the lever-arm effect, which arises when the slow roll variables evolve appreciably in the range of scales open to cosmological experimentation. Higher order slow roll consistency relations are automatically incorporated into slow roll reconstruction, and the significance of these terms grows rapidly as once increases the range of wavelengths over which we have information about the primordial spectrum. Our error forecasts for the slow roll parameters show that it is very unlikely this effect can be exploited by datasets containing only CMB information. However, high redshift $21 \mathrm{~cm}$ data may probe the the primordial spectrum at scales unreachable via the CMB. We thus conjecture that 
the combination of $\mathrm{CMB}$ and $21 \mathrm{~cm}$ data may test these consistency relations directly, fully realizing the power of slow roll reconstruction. This conjecture is supported by the recent analysis of [71], who project that $21 \mathrm{~cm}$ data could provide a $4 \sigma$ detection of the intrinsic running predicted by simple inflationary models. This is the same running one expects from higher-order consistency conditions, which is the basis of the lever arm effect we described earlier. There is thus good cause for optimism that slow roll reconstruction will be able to exploit this information to significantly tighten constraints on the inflationary parameter space and we plan to explore this issue in detail in a future publication. If inflation occurs well below the GUT scale, $\epsilon$ is very small, and the tensor modes are likely to be unobservable. The running in the spectral index induced by the scale dependence of the slow roll parameters is also proportional to $\epsilon$. Consequently, this effect is strongly correlated with the inflationary scale. Finally, BBO style direct detection experiments for gravitational waves probe the primordial spectrum at wavelengths some 30 e-folds smaller than those in the CMB [72] - and in this case the running of the spectral indices will be unmistakable if the tensor amplitude is large enough to be seen, and slow roll reconstruction will be well-placed to take advantage of these effects.

If the tensor to scalar ratio is vanishingly small, the tilt of the scalar spectrum is fixed by $\eta$, if $|\xi| \ll|\eta|$. If $\eta=-0.015$, corresponding to $n_{s}=0.97$, inflation lasts for much longer than 60 e-foldings. If our chosen set of variables provides a complete description of the spectrum, inflation must end via an some abrupt change in the potential, which cannot be encoded in the truncated slow roll expansion. Conversely, in models where $\epsilon$ is non-trivial, or the $\xi$ parameter is even slightly greater than zero, inflation will typically end within 60 e-folds, and often less. In this case, the inflaton can 'see' the end of inflation well before it occurs. Further, if $\epsilon$ is constrained to be very small by a tight upper bound on $r$, any running detected in the spectrum must be induced by an $\xi$ parameter, which is equivalent to learning that $V^{\prime \prime \prime}(\phi)$ is non-trivial.

In summary, this paper provides explores the theoretical basis of slow roll reconstruction, as originated by Easther and Peiris, and extends our understanding of the properties of the slow roll hierarchy. Slow roll reconstruction requires no explicit approximations in the treatment of the inflationary dynamics and exploits all possible sources of astrophysical information. Consequently it provides an optimal solution to the problem of reconstructing the inflationary potential from astrophysical data. Whether the universe will be kind enough to permit this program to be implemented in practice - and whether the generation of the primordial spectrum can in fact be attributed to an inflationary model which can be described in terms of a single, slowly-rolling field remains to be seen.

\section{Acknowledgments}

We thank Simeon Bird, Jan Hamann, Will Kinney, Julien Lesgourgues, Jonathan Pritchard,Wessel Valkenburg and Licia Verde for useful discussions, and acknowledge 
the use of the spectrum code of [17]. We are particularly indebted to Hiranya Peiris for providing the Hubble Slow Roll code, and for numerous discussions as this paper was written. PA and RE are supported in part by the United States Department of Energy, grant DE-FG02-92ER-40704. RE is supported by an NSF Career Award PHY-0747868.

\section{References}

[1] SDSS, M. Tegmark et al., Phys. Rev. D74, 123507 (2006), astro-ph/0608632.

[2] M. S. Turner, Phys. Rev. D48, 5539 (1993), astro-ph/9307035.

[3] E. J. Copeland, E. W. Kolb, A. R. Liddle, and J. E. Lidsey, Phys. Rev. D48, 2529 (1993), hep-ph/9303288.

[4] E. J. Copeland, E. W. Kolb, A. R. Liddle, and J. E. Lidsey, Phys. Rev. Lett. 71, 219 (1993), hep-ph/9304228.

[5] A. R. Liddle and M. S. Turner, Phys. Rev. D50, 758 (1994), astro-ph/9402021.

[6] H. Peiris and R. Easther, JCAP 0610, 017 (2006), astro-ph/0609003.

[7] H. Peiris and R. Easther, JCAP 0607, 002 (2006), astro-ph/0603587.

[8] R. Easther and H. Peiris, JCAP 0609, 010 (2006), astro-ph/0604214.

[9] M. B. Hoffman and M. S. Turner, Phys. Rev. D64, 023506 (2001), astro-ph/0006321.

[10] W. H. Kinney, Phys. Rev. D66, 083508 (2002), astro-ph/0206032.

[11] R. Easther and W. H. Kinney, Phys. Rev. D67, 043511 (2003), astro-ph/0210345.

[12] W. H. Kinney, E. W. Kolb, A. Melchiorri, and A. Riotto, Phys. Rev. D74, 023502 (2006), astro$\mathrm{ph} / 0605338$.

[13] B. A. Powell and W. H. Kinney, JCAP 0708, 006 (2007), arXiv:0706.1982 [astro-ph].

[14] J. E. Lidsey et al., Rev. Mod. Phys. 69, 373 (1997), astro-ph/9508078.

[15] A. R. Liddle and S. M. Leach, Phys. Rev. D68, 103503 (2003), astro-ph/0305263.

[16] A. R. Liddle, Phys. Rev. D68, 103504 (2003), astro-ph/0307286.

[17] J. Lesgourgues, A. A. Starobinsky, and W. Valkenburg, (2007), arXiv:0710.1630 [astro-ph].

[18] J. Hamann, J. Lesgourgues, and W. Valkenburg, (2008), arXiv:0802.0505 [astro-ph].

[19] S. M. Leach, A. R. Liddle, J. Martin, and D. J. Schwarz, Phys. Rev. D66, 023515 (2002), astro$\mathrm{ph} / 0202094$.

[20] S. M. Leach and A. R. Liddle, Mon. Not. Roy. Astron. Soc. 341, 1151 (2003), astro-ph/0207213.

[21] S. M. Leach and A. R. Liddle, Phys. Rev. D68, 123508 (2003), astro-ph/0306305.

[22] M. Malquarti, S. M. Leach, and A. R. Liddle, Phys. Rev. D69, 063505 (2004), astro-ph/0310498.

[23] J. M. Cline and L. Hoi, JCAP 0606, 007 (2006), astro-ph/0603403.

[24] R. Fisher, J. Roy. Stat. Soc. 98, 39 (1935).

[25] M. Tegmark, A. Taylor, and A. Heavens, Astrophys. J. 480, 22 (1997), astro-ph/9603021.

[26] A. Lewis, A. Challinor, and A. Lasenby, Astrophys. J. 538, 473 (2000), astro-ph/9911177.

[27] D. J. Eisenstein, W. Hu, and M. Tegmark, Astrophys. J. 518, 2 (1999), astro-ph/9807130.

[28] J. R. Bond, R. Crittenden, R. L. Davis, G. Efstathiou, and P. J. Steinhardt, Phys. Rev. Lett. 72, 13 (1994), astro-ph/9309041.

[29] L. Knox and M. S. Turner, Phys. Rev. Lett. 73, 3347 (1994), astro-ph/9407037.

[30] G. Jungman, M. Kamionkowski, A. Kosowsky, and D. N. Spergel, Phys. Rev. D54, 1332 (1996), astro-ph/9512139.

[31] M. Zaldarriaga and U. Seljak, Phys. Rev. D58, 023003 (1998), astro-ph/9803150.

[32] W. Hu and S. Dodelson, Ann. Rev. Astron. Astrophys. 40, 171 (2002), astro-ph/0110414.

[33] M. Zaldarriaga and U. Seljak, Phys. Rev. D55, 1830 (1997), astro-ph/9609170.

[34] J. R. Bond, G. Efstathiou, and M. Tegmark, Mon. Not. Roy. Astron. Soc. 291, L33 (1997), astro-ph/9702100.

[35] Y. Wang, D. N. Spergel, and M. A. Strauss, Astrophys. J. 510, 20 (1999), astro-ph/9802231. 
[36] M. McQuinn, O. Zahn, M. Zaldarriaga, L. Hernquist, and S. R. Furlanetto, Astrophys. J. 653, 815 (2006), astro-ph/0512263.

[37] E. J. Copeland, E. W. Kolb, A. R. Liddle, and J. E. Lidsey, Phys. Rev. D49, 1840 (1994), astro-ph/9308044.

[38] A. Kosowsky and M. S. Turner, Phys. Rev. D52, 1739 (1995), astro-ph/9504071.

[39] L. Grishchuk and Y. V. Sidorav, Fourth Seminar on Quantum Gravity, World Scientific, Singapore.

[40] A. G. Muslimov, Class. Quant. Grav. 7, 231 (1990).

[41] D. S. Salopek and J. R. Bond, Phys. Rev. D42, 3936 (1990).

[42] D. S. Salopek and J. R. Bond, Phys. Rev. D43, 1005 (1991).

[43] J. E. Lidsey, Phys. Lett. B273, 42 (1991).

[44] G. Ballesteros, J. A. Casas, and J. R. Espinosa, JCAP 0603, 001 (2006), hep-ph/0601134.

[45] V. F. Mukhanov, H. A. Feldman, and R. H. Brandenberger, Phys. Rept. 215, 203 (1992).

[46] E. D. Stewart and D. H. Lyth, Phys. Lett. B302, 171 (1993), gr-qc/9302019.

[47] J. A. Adams, B. Cresswell, and R. Easther, Phys. Rev. D64, 123514 (2001), astro-ph/0102236.

[48] C. Ringeval, Lect. Notes Phys. 738, 243 (2008), astro-ph/0703486.

[49] D. S. Salopek, J. R. Bond, and J. M. Bardeen, Phys. Rev. D40, 1753 (1989).

[50] WMAP, H. V. Peiris et al., Astrophys. J. Suppl. 148, 213 (2003), astro-ph/0302225.

[51] WMAP, D. N. Spergel et al., (2006), astro-ph/0603449.

[52] C. L. Reichardt et al., (2008), arXiv:0801.1491 [astro-ph].

[53] A. D. Linde, Phys. Rev. D49, 748 (1994), astro-ph/9307002.

[54] E. J. Copeland, A. R. Liddle, D. H. Lyth, E. D. Stewart, and D. Wands, Phys. Rev. D49, 6410 (1994), astro-ph/9401011.

[55] A. R. Liddle and D. H. Lyth, Cosmological Inflation and Large-Scale Structure (Cambridge University Press, 2000).

[56] W. H. Kinney and A. Riotto, JCAP 0603, 011 (2006), astro-ph/0511127.

[57] D. J. H. Chung, L. L. Everett, and K. T. Matchev, Phys. Rev. D76, 103530 (2007), arXiv:0704.3285 [hep-ph].

[58] C. P. Burgess, R. Easther, A. Mazumdar, D. F. Mota, and T. Multamaki, JHEP 05, 067 (2005), hep-th/0501125.

[59] L. McAllister and E. Silverstein, (2007), arXiv:0710.2951 [hep-th].

[60] L. A. Boyle, P. J. Steinhardt, and N. Turok, Phys. Rev. Lett. 96, 111301 (2006), astro-ph/0507455.

[61] D. H. Lyth, Phys. Rev. Lett. 78, 1861 (1997), hep-ph/9606387.

[62] L. Verde, H. Peiris, and R. Jimenez, JCAP 0601, 019 (2006), astro-ph/0506036.

[63] the Clover, A. C. Taylor, New Astron. Rev. 50, 993 (2006), astro-ph/0610716.

[64] J. Bock et al., (2006), astro-ph/0604101.

[65] L. Knox and Y.-S. Song, Phys. Rev. Lett. 89, 011303 (2002), astro-ph/0202286.

[66] R. Easther, W. H. Kinney, and B. A. Powell, JCAP 0608, 004 (2006), astro-ph/0601276.

[67] W. H. Kinney, Phys. Rev. D58, 123506 (1998), astro-ph/9806259.

[68] A. Albrecht et al., (2006), astro-ph/0609591.

[69] S. Chongchitnan and G. Efstathiou, Phys. Rev. D72, 083520 (2005), astro-ph/0508355.

[70] J. Hamann, S. Hannestad, M. S. Sloth, and Y. Y. Y. Wong, Phys. Rev. D75, 023522 (2007), astro-ph/0611582.

[71] Y. Mao, M. Tegmark, M. McQuinn, M. Zaldarriaga, and O. Zahn, (2008), arXiv:0802.1710 [astro$\mathrm{ph}]$.

[72] T. L. Smith, H. V. Peiris, and A. Cooray, Phys. Rev. D73, 123503 (2006), astro-ph/0602137.

\section{Appendix A. Solutions to the Slow Roll Hierarchy}

High- $\epsilon$ 1-Parameter: The case where we truncate the flow equations at first order corresponds to a potential that is quadratic, at least around the pivot. We can solve 
the flow equations exactly in this case, irrespective of the value of $\epsilon$ :

$$
\epsilon(N)=\frac{\epsilon_{0}}{2 N \epsilon_{0}+1},
$$

where $\epsilon_{0}$ is the value of $\epsilon$ at the fiducial scale (where we have set $N=0$ ). We find that inflation ends after

$$
N_{\text {end }}=\frac{1-\epsilon_{0}}{2 \epsilon_{0}} .
$$

The value of $\epsilon$ can be tuned to obtain the right amount of inflation to satisfy the matching condition, equation (25). However, as we have discussed above, in a one parameter model, $\epsilon$ is tightly constrained by the tilt, tensor-scalar ratio and tensor tilt. Any tension between these quantities will result in the need for non-zero higher order HSR parameters.

Low- $\epsilon$ 1-Parameter: See section 3 for details of this case.

Low- $\epsilon$ 2-Parameter: In this situation, the third flow equation is

$$
\frac{d \xi}{d N}=\eta \xi
$$

In the limit $\epsilon \ll 1$ we can solve equations (26) and (A.3) to obtain

$$
\begin{aligned}
& \eta(N)=-\sqrt{2 A_{\xi}} \tan \left[\frac{1}{2} \sqrt{2 A_{\xi}}\left(2 B_{\eta}-N\right)\right], \\
& \xi(N)=A_{\xi} \tan ^{2}\left[\frac{1}{2} \sqrt{2 A_{\xi}}\left(2 B_{\eta}-N\right)\right]+A_{\xi},
\end{aligned}
$$

where $A_{\xi}=\xi_{0}-\frac{1}{2} \eta_{0}^{2}$ and $B_{\eta}=\arctan \left(-\eta_{0} / \sqrt{2 A_{\xi}}\right) / \sqrt{2 A_{\xi}}$. Finally,

$$
\epsilon(N)=C_{\epsilon} \sec \left[\frac{1}{2} \sqrt{2 A_{\xi}}\left(2 B_{\eta}-N\right)\right]^{4},
$$

where $C_{\epsilon}=\epsilon_{0} / \sec ^{4}\left(\sqrt{2 A_{\xi}} B_{\eta}\right)$. We can now solve for where inflation ends (where $\epsilon=1$ ) in this approximation:

$$
-N=\frac{2 \arccos \left(C_{\epsilon}^{1 / 4}\right)}{\sqrt{2 A_{\xi}}}-2 B_{\eta}
$$

The upper panels of figure A1 show the quality of the approximation, which only gets better as one decreases the value of $\epsilon$. The lower panels of figure A1 shows the number of e-folds as a function of the slow roll parameters $\eta$ and $\xi$ and the tilt and running of the scalar spectral index respectively. The end of inflation in these models is completely specified by the initial values of the slow roll parameters.

Using a value of $\xi$ that gives a running anywhere near the centroid of the WMAP data [51] leads to a short period of inflation. If the current weak evidence for running detected in the WMAP data persists, higher order slow roll parameters are required to produce enough inflation. This is consistent with the findings of [8]. 

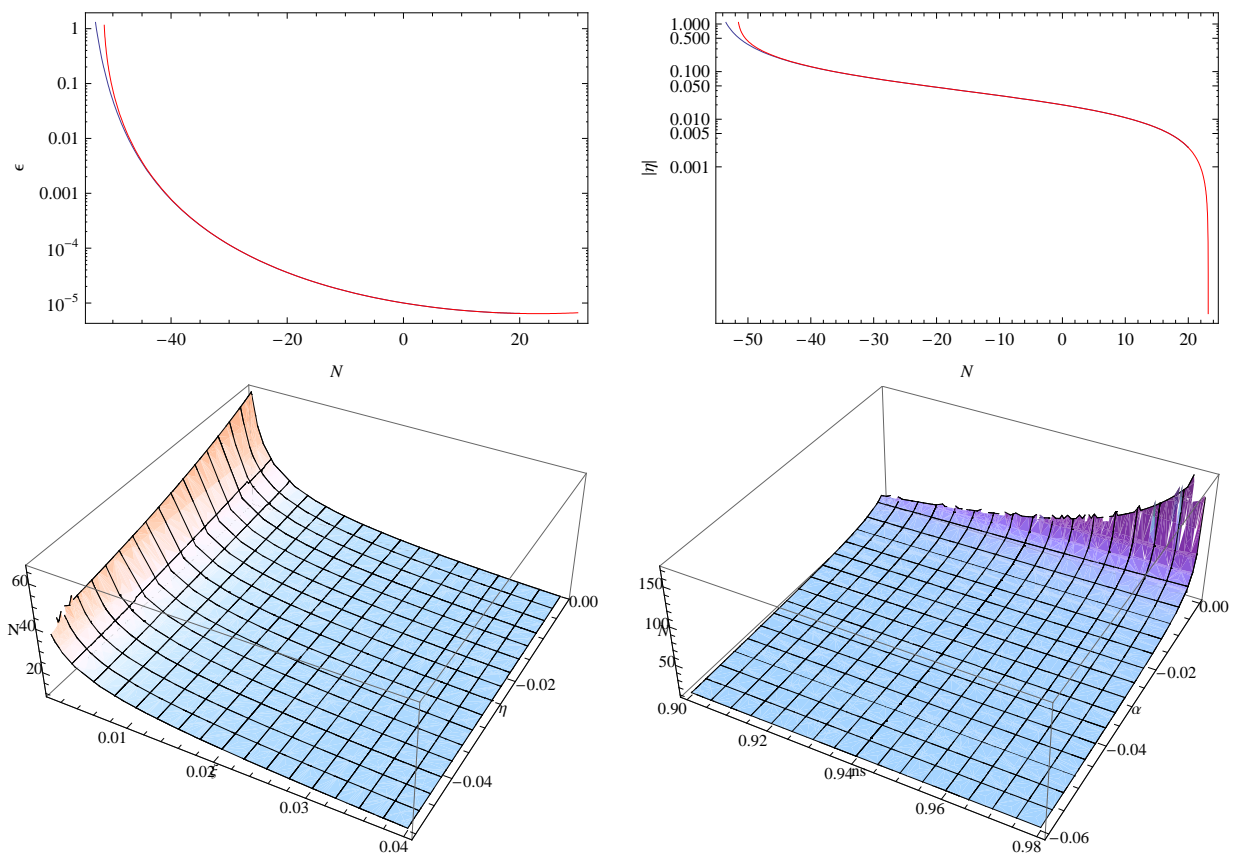

Figure A1. Slow roll hierarchy truncated at third order $\left({ }^{3} \lambda_{H}=0\right)$ and $\xi=0.001$. The left panel shows the evolution of $\epsilon$ while the right panel show $\eta$; the red curve is the exact result, the blue is the approximation. The lower left plot shows the amount of inflation as a function of the parameters $\eta$ and $\xi$, while the lower right plot shows the same quantity as a function of the spectral indices.
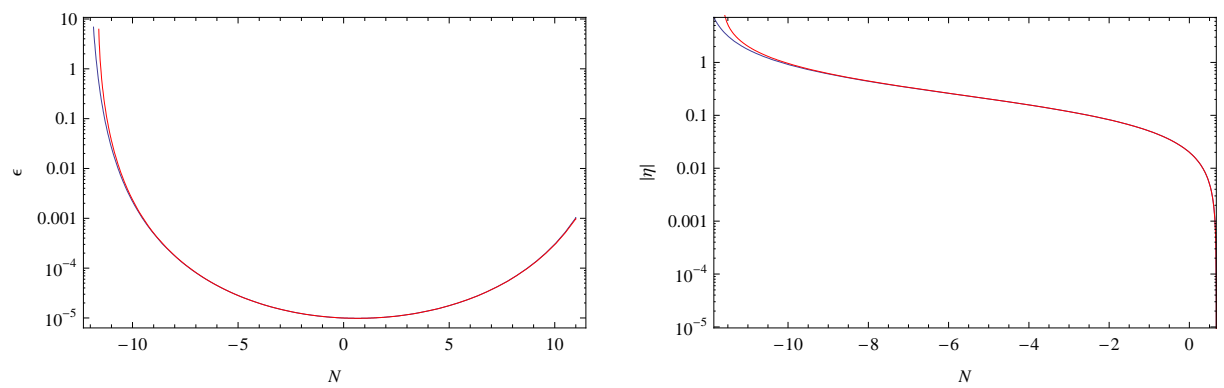

Figure A2. Slow roll hierarchy truncated at fourth order ${ }^{4} \lambda_{H}=0$. At the pivot, $\eta=-0.02, \xi=0.03,{ }^{3} \lambda_{H}=-0.0001$ and $\epsilon=10^{-5}$. Left panel show the evolution of $\epsilon$, the red curve is the exact result, the blue is the approximation.

Low- $\epsilon$ 3-Parameter If we include ${ }^{3} \lambda_{H}$ there is no obvious analytic solution. However, if ${ }^{3} \lambda_{H}$ is small, and noting that $\eta$ is also small and remains small throughout inflation, we employ a further approximation:

$$
\frac{d^{3} \lambda_{H}}{d N} \approx 0
$$

In this limit $\eta$ obeys Airy's equation, with solution:

$$
\eta(N)=-\left(\frac{ \pm^{3} \lambda_{H}}{2}\right)^{1 / 3}\left(\frac{C_{1} \mathrm{Ai}^{\prime}[\mathrm{x}(\mathrm{N})]+\mathrm{Bi}^{\prime}[\mathrm{x}(\mathrm{N})]}{C_{1} \mathrm{Ai}[\mathrm{x}(\mathrm{N})]+\mathrm{Bi}[\mathrm{x}(\mathrm{N})]}\right)
$$


where

$$
x(N)=\mp\left(\frac{ \pm^{3} \lambda_{H}}{2}\right)^{1 / 3}\left(N+\frac{A_{\xi}}{{ }^{3} \lambda_{H}}\right),
$$

and $C_{1}$ is a constant given by:

$$
C_{1}=-\left(\frac{\mathrm{Bi}^{\prime}[\mathrm{x}(0)]+\left(\frac{ \pm^{3} \lambda_{\mathrm{H}}}{2}\right)^{-1 / 3} \eta_{0} \mathrm{Bi}[\mathrm{x}(0)]}{\mathrm{Ai}^{\prime}[\mathrm{x}(0)]+\left(\frac{ \pm^{3} \lambda_{\mathrm{H}}}{2}\right)^{-1 / 3} \eta_{0} \mathrm{Ai}[\mathrm{x}(0)]}\right) .
$$

Now we can also solve for $\epsilon(N)$ to obtain

$$
\epsilon(N)=\frac{C_{2}}{\left(C_{1} \operatorname{Ai}[\mathrm{x}(\mathrm{N})]+\mathrm{Bi}[\mathrm{x}(\mathrm{N})]\right)^{4}}
$$

where $C_{2}$ is given by:

$$
\mathrm{C}_{2}=\epsilon_{0}\left(\mathrm{C}_{1} \mathrm{Ai}[\mathrm{x}(0)]+\mathrm{Bi}[\mathrm{x}(0)]\right)^{4}
$$

Figure A2 shows the quality of the approximation, even for large values of the running, or $\xi$. The value of $\xi$ here is taken to coincide with a value of $\alpha=-0.06$, i.e. $\xi=0.03$. Notice how quickly inflation ends; barely 10 e-folds of inflation are achieved.

These expressions are of limited use in practice. If ${ }^{3} \lambda_{H}$ is large enough to prevent $\xi$ from dominating the dynamics, it must be of the same magnitude as $\xi$, and equation (A.7) is no longer a good approximation. The effect of including ${ }^{3} \lambda_{H}$ depend on its sign. If ${ }^{3} \lambda_{H}<0$, inflation ends more rapidly, for fixed $\{\epsilon, \eta, \xi\}$. If $0<{ }^{3} \lambda_{H} \ll 1$, we see a slight increase in the number of e-folds. As one increases ${ }^{3} \lambda_{H}$, one encounters a critical point is reached where $\epsilon$ has a maximal value less than unity and then begins to decrease. 